\title{
An asymptotic expansion for the vortex-induced vibrations of a circular cylinder
}

\author{
PHILIPPE MELIGA†AND JEAN-MARC CHOMAZ
}

LadHyX, CNRS-Ecole Polytechnique, 91128 Palaiseau, France

(Received 4 February 2010; revised 20 July 2010; accepted 15 October 2010; first published online 17 February 2011)

This paper investigates the vortex-induced vibrations (VIV) of a spring-mounted circular cylinder. We compute analytically the leading-order equations describing the nonlinear interaction of the fluid and structure modes by carrying out an asymptotic analysis of the Navier-Stokes equations close to the threshold of instability of the fluid-only system. We show that vortex-shedding can occur at subcritical Reynolds numbers as a result of the coupled system being linearly unstable to the structure mode. We also show that resonance occurs when the frequency of the nonlinear limit cycle matches the natural frequency of the cylinder, the displacement being then in phase with the flow-induced lift fluctuations. Using an extension of this model meant to encompass the effect of the low-order added-mass and damping forces induced by the displaced fluid, we show that the amount of energy that can be extracted from the flow can be optimized by an appropriate choice of the structural parameters. Finally, we suggest a possible connection between the present 'exact' model and the empirical wake oscillator model used to study VIV at high Reynolds numbers. We show that for the low Reynolds numbers considered here, the effect of the structure on the fluid can be represented by a first coupling term proportional to the cylinder acceleration in the fluid equation, and by a second term of lower magnitude, which can stem either from an integral term or from a term proportional to the third derivative of the cylinder position.

Key words: flow-structure interactions, instability, nonlinear dynamical systems

\section{Introduction}

Numerous studies have been devoted to the problem of the flow past a fixed cylinder. It is now well established that the flow undergoes a global instability at a critical Reynolds number, $R e_{*} \sim 47$, responsible for the onset of the time-periodic vortexshedding phenomenon (Mathis, Provansal \& Boyer 1984; Provansal, Mathis \& Boyer 1987). This causes the cylinder to experience unsteady lift and drag forces. If mounted on elastic supports, the cylinder should then undergo vortex-induced vibrations (VIV); see Williamson \& Govardhan (2004), Sarpkaya (2004) and Gabbai \& Benaroya (2005) for a review of the recent progress achieved in this field.

Most studies about VIV of bluff bodies have been carried out at high Reynolds numbers, where one usually has to resort to some empirical modelling of the wake dynamics (Sarpkaya 1978; Blevins 1990). A restricted number of studies have been devoted to the onset of VIV near the threshold of instability. Cossu \& Morino 
(2000) have carried out a linear global stability analysis of the flow past a springmounted cylinder and have identified two modes of interest: the nearly structural mode, renamed here structure mode, and the von Kármán mode, renamed here wake mode. These modes correspond to eigenvalues which, in the limit of very large solidto-fluid density ratios (i.e. very weak fluid-structure interaction), tend respectively to the natural eigenvalue of the cylinder-only system and to the leading eigenvalue that can be computed from the flow past a fixed cylinder. These authors have also shown that vortex shedding can persist at subcritical conditions for low-density ratios (i.e. strong fluid-structure interaction), and have provided evidence connecting this phenomenon to an instability of the structure mode. Such a destabilization has since then been confirmed by the experimental observations of Buffoni (2003) and the numerical simulations of Mittal \& Singh (2005).

The first objective of the present work is to investigate VIV close to the critical Reynolds number $R e_{*}$ by combining asymptotic analyses and adjoint-based receptivity methods. The main advantage of this approach, which is valid rigorously for small departures from the instability threshold and small cylinder displacements, lies in the fact that it defines a dominant balance justifying to treat the coupling between the flow and the cylinder motions implicitly. This coupling results from a compatibility condition applied to an expansion where the cylinder is fixed at leading-order, so that no specific treatment is required in the numerics (for instance mesh deformation schemes, as discussed by Tezduyar, Behr \& Liou $1992 a$ and Tezduyar et al. 1992b). Such an approach also provides additional insight into the flow physics by giving access to the second-order solution, which is essential when discussing some realistic features of the coupled system, for instance its ability to sustain complex hysteretic behaviours in the lock-in regime (Williamson \& Govardhan 2004). The second objective is to establish a connection between the present bifurcation theory based on the Navier-Stokes equations and the wake oscillator model widely used for the study of VIV at high Reynolds numbers (see Parkinson 1989; Blevins 1990, for a review).

This paper is organized as follows. The configuration under study is presented in $\S 2$. The flow and structure models are discussed in $\S 3$, where we detail the asymptotic expansion used to derive the coupled model. The linear and nonlinear dynamics are investigated in $\S \S 4-5$, where the effect of the structural parameters is discussed. In $\S 6$, we propose an extension of the model meant for low-density ratios and mass-damping parameters, where one must take into account added-mass and added damping forces induced by the mass of displaced fluid. In $\S 7$, we use the extended model to assess the influence of the structural damping on the amount of energy that can be extracted from the flow and dissipated by the structure, which is of practical interest when VIV are thought to be used for energy production. Section 8 is ultimately devoted to the connection between the present model and the wake oscillator model.

\section{Configuration}

We investigate the dynamics of a rigid circular cylinder of surface $\Upsilon_{w}$, immersed in a uniform flow, as illustrated in figure 1. The fluid is homogeneous and incompressible, of density $\rho$ and kinematic viscosity $\nu$. In the following, all quantities are made non-dimensional using the cylinder diameter $2 R$ and the free-stream velocity $u_{\infty}$. The Cartesian coordinate system $\left(0, \boldsymbol{e}_{x}, \boldsymbol{e}_{y}\right)$, with $\boldsymbol{e}_{x}$ and $\boldsymbol{e}_{y}$ being the streamwise and transverse directions, has its origin taken at the centre of the cylinder. The fluid motion is described by the velocity field $\boldsymbol{u}=(u, v)^{\mathrm{T}}$ ( $u$ and $v$ being the streamwise and transverse components) and the pressure field $p$. The state vector $\boldsymbol{q}=(\boldsymbol{u}, p)^{\mathrm{T}}$ obeys 

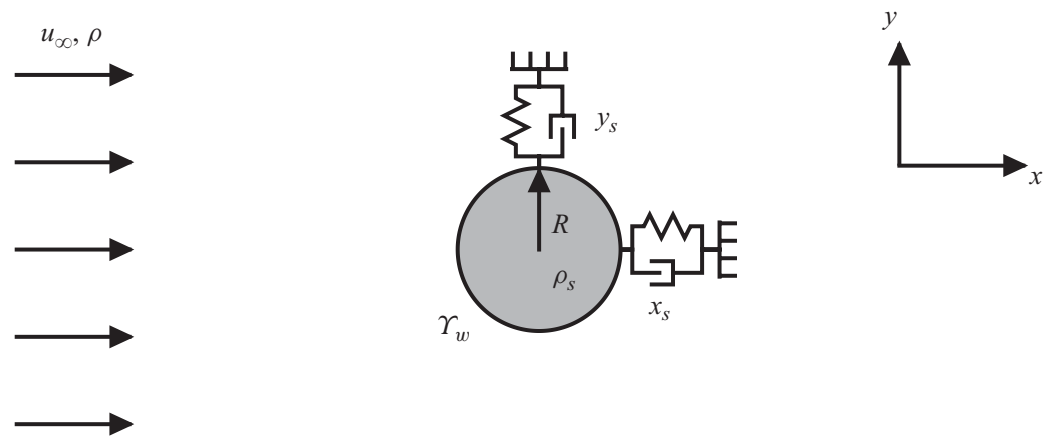

FIGURE 1. Schematic diagram of the spring-mounted, damped cylinder configuration.

the incompressible Navier-Stokes equations

$$
\nabla \cdot \boldsymbol{u}=0, \quad \frac{\partial \boldsymbol{u}}{\partial t}+\nabla \boldsymbol{u} \cdot \boldsymbol{u}+\nabla p-\frac{1}{R e} \nabla^{2} \boldsymbol{u}=0,
$$

written formally as

$$
\boldsymbol{N} \frac{\partial \boldsymbol{q}}{\partial t}+\boldsymbol{M}(\boldsymbol{q}, R e)=\mathbf{0}
$$

with $R e$ being the Reynolds number and $\boldsymbol{N}$ and $\boldsymbol{M}$ being differential operators.

The cylinder is mounted on springs of identical stiffness in the streamwise and transverse directions, its displacement being a simple translation with no rotation. It is subjected to external damping and to the action of the fluid, so that its motion is governed by the linear oscillator equations

$$
\frac{\mathrm{d}^{2} x_{s}}{\mathrm{~d} t^{2}}+2 \omega_{s} \gamma \frac{\mathrm{d} x_{s}}{\mathrm{~d} t}+\omega_{s}^{2} x_{s}=\frac{2}{\pi m} c_{x}, \quad \frac{\mathrm{d}^{2} y_{s}}{\mathrm{~d} t^{2}}+2 \omega_{s} \gamma \frac{\mathrm{d} y_{s}}{\mathrm{~d} t}+\omega_{s}^{2} y_{s}=\frac{2}{\pi m} c_{y},
$$

where $x_{s}$ and $y_{s}$ stand for the non-dimensional streamwise and transverse displacements of the cylinder centre. In (2.3), $\omega_{s}$ is the dimensionless natural frequency in vacuum, $\gamma$ is the structural damping coefficient, and $m$ is the mass ratio defined as the ratio of the solid to the fluid density, i.e. $m=\rho_{s} / \rho$. Likewise, $c_{x}$ is the instantaneous drag coefficient of the cylinder per unit length, defined as

$$
c_{x}=2 \int_{\Upsilon_{w}}\left(-p \boldsymbol{n}+\frac{1}{R e}\left(\nabla \boldsymbol{u}+\nabla \boldsymbol{u}^{\mathrm{T}}\right) \cdot \boldsymbol{n}\right) \cdot \boldsymbol{e}_{x} \mathrm{~d} l,
$$

with $n$ being the outward-pointing vector normal to the cylinder and $\mathrm{d} l$ being the length element along $\Upsilon_{w}$. Finally, $c_{y}$ is the instantaneous lift coefficient defined similarly by substituting $\boldsymbol{e}_{x}$ by $\boldsymbol{e}_{y}$ in (2.4).

On the cylinder wall, we enforce that the fluid velocity differs from that of the cylinder only when a blowing and suction control velocity $\boldsymbol{u}_{\boldsymbol{c}}$ is applied:

$$
\boldsymbol{u}\left(x+x_{s}, y+y_{s}\right)=\boldsymbol{u}_{c}\left(x+x_{s}, y+y_{s}\right)+\left(\frac{\mathrm{d} x_{s}}{\mathrm{~d} t}, \frac{\mathrm{d} y_{s}}{\mathrm{~d} t}\right)^{\mathrm{T}}, \quad \forall(x, y) \in \Upsilon_{w},
$$

all quantities being evaluated at the boundary of the moving cylinder.

\section{Asymptotic expansion}

The genesis of vortex shedding past a fixed circular cylinder has been widely studied in the framework of the global stability theory, in which one characterizes the stability 
of the steady solution to small-amplitude perturbations $\boldsymbol{q}_{1}$, expanded as

$$
\boldsymbol{q}_{1}(x, y, t)=\hat{\boldsymbol{q}}_{1}(x, y) \mathrm{e}^{(\sigma+\mathrm{i} \omega) t}+\text { c.c. }
$$

In (3.1), $\sigma$ and $\omega$ are the growth rate and pulsation of the global eigenmode $\hat{\boldsymbol{q}}_{1}$, and c.c. denotes the complex conjugate of the preceding expression. For Reynolds numbers $R e \geqslant R e_{*}$, the steady flow becomes unstable to a global mode of marginal pulsation $\omega_{*} \sim 0.74$, corresponding to a Strouhal number $S t_{*}=\omega_{*} / 2 \pi \sim 0.118$. Its eigenvector $\hat{\boldsymbol{q}}_{1 A}$ is a solution of the generalized eigenvalue problem

$$
\left(\mathrm{i} \omega_{*} \boldsymbol{N}+\boldsymbol{L}_{*}\right) \hat{\boldsymbol{q}}_{1 A}=\mathbf{0},
$$

$\boldsymbol{L}_{*}$ being the Navier-Stokes operator linearized around $\boldsymbol{q}_{0}$ at the critical Reynolds number (Jackson 1987; Barkley 2006; Giannetti \& Luchini 2007; Sipp \& Lebedev 2007).

We investigate here the coupled flow-structure system by means of a standard multiple time-scale analysis. We introduce the fast time scale $t$ and the slow time scale $T=\epsilon^{2} t$ and assume that $R e$ departs from criticality at order $\epsilon^{2}$. We thus define the order-one parameter $\delta$, such that

$$
\frac{1}{R e}=\frac{1}{R e_{*}}-\epsilon^{2} \delta,
$$

and expand the flow field $\boldsymbol{q}$ as

$$
\boldsymbol{q}=\boldsymbol{q}_{0}+\epsilon \boldsymbol{q}_{1}+\epsilon^{2} \boldsymbol{q}_{2}+\epsilon^{3} \boldsymbol{q}_{3}+\cdots .
$$

Since the steady solution is symmetric with respect to the $y=0$ axis, i.e. it satisfies $(u, v, p) \longrightarrow(u,-v, p)$ under the $y \rightarrow-y$ reflection, it exerts a pure drag force on the cylinder, whose centre reaches an equilibrium position defined by

$$
\omega_{s}^{2} x_{s}^{0}=\frac{2}{\pi m} c_{x}^{0}, \quad y_{s}^{0}=0 .
$$

It can be anticipated from the dominant balance that a small-amplitude cylinder displacement at order $\epsilon^{3}$ around this equilibrium position is sufficient to force the global mode at order $\epsilon$ when forcing is near-resonance. The displacement is therefore written as

$$
x_{s}-x_{s}^{0}=\epsilon^{3} X(T) \mathrm{e}^{\mathrm{i} \omega_{s} t}+\text { c.c., } \quad y_{s}-y_{s}^{0}=\epsilon^{3} Y(T) \mathrm{e}^{\mathrm{i} \omega_{s} t}+\text { c.c. },
$$

where the two complex amplitudes $X$ and $Y$ are at this stage unknown functions of the slow time $T$. This third-order scaling allows to decouple the flow and cylinder dynamics at leading-order $\epsilon$. It brings the forcing of the flow motion by the cylinder displacement at the same order as the linear growth owing to the departure from threshold and the resonant nonlinear terms, both being expected to occur on a time scale of order $1 / \epsilon^{2}$, as would be the case if the cylinder were fixed. If the displacement were chosen of larger order, both the flow and cylinder dynamics would be coupled at leading order and the instability modes would have to be directly computed, as in Cossu \& Morino (2000). For the same reason, the control velocity at the cylinder wall is also chosen of order $\epsilon^{3}$ :

$$
\boldsymbol{u}_{\boldsymbol{c}}=\epsilon^{3} \boldsymbol{U}_{\boldsymbol{c}}(T) \mathrm{e}^{\mathrm{i} \omega_{s} t}+\text { c.c. }
$$


Substitution of the preceding expansions into (2.5) yields the series of boundary conditions at the cylinder wall $\Upsilon_{w}$ :

$$
\begin{aligned}
& \boldsymbol{u}_{\boldsymbol{i}}=\mathbf{0}(i=0, \ldots, 2), \\
& \boldsymbol{u}_{3}=\left(\boldsymbol{U}_{\boldsymbol{w}}(T)+\boldsymbol{U}_{\boldsymbol{c}}(T)\right) \mathrm{e}^{\mathrm{i} \omega_{*} t}+\text { c.c., } \quad \text { with } \boldsymbol{U}_{\boldsymbol{w}}=\left(\mathrm{i} \omega_{*} \boldsymbol{I}-\nabla \boldsymbol{u}_{0}\right) \cdot(X, Y)^{\mathrm{T}} .
\end{aligned}
$$

Owing to the present balance, the flow problem is that of the wake past a fixed cylinder, up to the second order $\epsilon^{2}$, meaning that, at this stage, no specific treatment is required in the numerics to take into account the moving boundary. Coupling arises at order $\epsilon^{3}$, as the flow motion is forced by an equivalent resonant blowing and suction velocity that can be split into two distinct components issuing respectively from the cylinder displacement and from the imposed control velocity, all quantities in $(3.8 b)$ being evaluated at the boundary of the fixed cylinder.

Since the order $\epsilon^{3}$ cylinder displacement is forced near-resonance, and because the flow-induced drag and lift fluctuations are proportional to the global mode amplitude of order $\epsilon$, we again anticipate from the dominant balance by imposing a large mass ratio of order $\epsilon^{-4}$, i.e. such that

$$
\frac{1}{m}=\epsilon^{4} \frac{1}{M},
$$

where $M$ is the rescaled mass ratio of order one. In practice, this fourth-order scaling is legitimate for steel in air, for which the assumption of a rigid cylinder with no deformation holds. It is meant for the inertial and fluctuating forces to be in balance in the oscillator equations (2.3), and it can be indeed checked that any larger order would only yield trivial solutions in which the cylinder would either remain stationary or be slaved to the lift fluctuations. Similarly, we set the detuning between the natural and marginal frequencies and the structural damping at second order, for the restoring and damping forces to be in balance with the inertial one in (2.3). This yields

$$
\omega_{s}=\omega_{*}\left(1+\epsilon^{2} \Omega\right), \quad \gamma=\epsilon^{2} \Gamma,
$$

where $\Omega$ is the rescaled detuning parameter and $\Gamma$ is the rescaled damping parameter, both quantities being of order one.

The key non-trivial dominant balance is thus to assume a large mass ratio of order $1 / \epsilon^{4}$, at the same time as small departure from criticality, detuning and structural damping of order $\epsilon^{2}$. Both the global mode $\hat{\boldsymbol{q}}_{1 A}$ and the cylinder displacement are forced close to resonance and amplify their respective forcing by a factor of $1 / \epsilon^{2}$, thus yielding a strong coupling. This dominant balance is meant to set all possible effects at the same order, meaning that the set of coupled equations derived in the following is the most general possible.

Note that it is possible to express the reduced flow velocity $u_{r}$ classically used in the literature as

$$
u_{r}=\frac{2 \pi}{\omega_{s}}=\frac{1}{S t_{*}\left(1+\epsilon^{2} \Omega\right)} .
$$

At leading order, this yields $u_{r} \sim\left(1-\epsilon^{2} \Omega\right) / S t_{*}$, so that $\Omega$ can also be understood as a measure of the departure from the reference reduced velocity defined as $1 / S t_{*}$. Still, in the following, results are presented in terms of $1 / u_{r}$ in order to keep the generality of the problem. 


\subsection{Flow model}

At order $\epsilon^{0}$, the solution $\boldsymbol{q}_{0}$ is the steady flow developing past a fixed cylinder, solution of the nonlinear equations

$$
\boldsymbol{M}\left(\boldsymbol{q}_{0}, R e_{*}\right)=\mathbf{0} .
$$

The equations at order $\epsilon$ are the linearized Navier-Stokes equations that define $\boldsymbol{q}_{1}$ as a superposition of global modes developing on $\boldsymbol{q}_{0}$. It can therefore be chosen as the marginally stable mode $\hat{\boldsymbol{q}}_{1 A}$ multiplied by some unknown complex amplitude $A$, depending only on the slow time $T$, i.e.

$$
\boldsymbol{q}_{1}=A(T) \hat{\boldsymbol{q}}_{1 A} \mathrm{e}^{\mathrm{i} \omega_{*} t}+\text { c.c. }
$$

The second-order solution $\boldsymbol{q}_{2}$ can be computed as

$$
\boldsymbol{q}_{2}=\delta \hat{\boldsymbol{q}}_{2 \delta}+|A|^{2} \hat{\boldsymbol{q}}_{2|A|^{2}}+\left(A^{2} \hat{\boldsymbol{q}}_{2 A^{2}} \mathrm{e}^{2 \mathrm{i} \omega_{*} t}+\text { c.c. }\right) \text {. }
$$

In (3.14), $\hat{\boldsymbol{q}}_{2 \delta}, \hat{\boldsymbol{q}}_{2|A|^{2}}$ and $\hat{\boldsymbol{q}}_{2 A^{2}}$ are solutions of the forced linear problems

$$
\begin{aligned}
\boldsymbol{L}_{*} \hat{\boldsymbol{q}}_{2 \delta} & =\left(-\nabla^{2} \boldsymbol{u}_{0}, 0\right)^{\mathrm{T}}, \\
\boldsymbol{L}_{*} \hat{\boldsymbol{q}}_{2|A|^{2}} & =\left(-\nabla \hat{\boldsymbol{u}}_{1 A} \cdot \overline{\hat{\boldsymbol{u}}_{1 A}}-\overline{\nabla \hat{\boldsymbol{u}}_{1 A}} \cdot \hat{\boldsymbol{u}}_{1 A}, 0\right)^{\mathrm{T}}, \\
\left(2 \mathrm{i} \omega_{*} \boldsymbol{N}+\boldsymbol{L}_{*}\right) \hat{\boldsymbol{q}}_{2 A^{2}} & =\left(-\nabla \hat{\boldsymbol{u}}_{1 A} \cdot \hat{\boldsymbol{u}}_{1 A}, 0\right)^{\mathrm{T}},
\end{aligned}
$$

where the overbars indicate complex conjugate quantities. The first forcing term on the right-hand side of $(3.15 a)$ is linear and arises from the change in the diffusion of the base flow when the Reynolds number departs from threshold. The other two terms on the right-hand sides of $(3.15 b)$ and $(3.15 c)$ are nonlinear and arise from the advection of the first-order solution by itself, which yields both a steady and a harmonic forcing. Physically, $\hat{\boldsymbol{q}}_{2 \delta}$ therefore represents the modification of the base flow observed when the Reynolds number is varied, $\hat{\boldsymbol{q}}_{2|A|^{2}}$ is the difference between the mean flow and the base flow, whereas $\hat{\boldsymbol{q}}_{2 A^{2}}$ is the second-order harmonic contribution (Sipp \& Lebedev 2007). Since 0 and $2 i \omega_{*}$ are not eigenvalues for the fixed cylinder flow at the critical Reynolds number $R e_{*}$, none of these forcing terms are resonant, meaning that (3.15) can be solved numerically by carrying out a simple matrix inversion.

In contrast, a compatibility condition has to be enforced at order $\epsilon^{3}$, where resonant forcing terms of frequency $\omega_{*}$ set in. This requires to compute the adjoint global mode $\hat{\boldsymbol{q}}_{1 A}^{\dagger}$ solution of the adjoint eigenvalue problem

$$
\left(-\mathrm{i} \omega_{*} \boldsymbol{N}+\boldsymbol{L}_{*}^{\dagger}\right) \hat{\boldsymbol{q}}_{1 A}^{\dagger}=\mathbf{0}
$$

where $\boldsymbol{L}_{*}^{\dagger}$ is the adjoint of operator $\boldsymbol{L}_{*}$, obtained by integrating by parts system (3.2) (see Schmid \& Henningson 2001 for details). Owing to the present balance, the flow-cylinder coupling directly adds on to the third-order resonant terms through the resonant boundary condition $(3.8 b)$. All details of the procedure are provided in Appendix B. In particular, we show that the compatibility condition imposes the amplitudes $A, X, Y$ to obey the relation

$$
\frac{\mathrm{d} A}{\mathrm{~d} T}=\lambda \delta A-\mu A|A|^{2}+\alpha_{x} X+\alpha_{y} Y+\eta .
$$

In (3.17), $\lambda$ and $\mu$ are the complex coefficients defined by

$$
\lambda=\mathscr{S}^{-1} \int_{\Sigma} \hat{\boldsymbol{q}}_{1 A}^{\dagger} \cdot \hat{\boldsymbol{F}}_{3 A} \mathrm{~d} x \mathrm{~d} y, \quad \mu=-\mathscr{S}^{-1} \int_{\Sigma} \hat{\boldsymbol{q}}_{1 A}^{\dagger} \cdot \hat{\boldsymbol{F}}_{3 A|A|^{2}} \mathrm{~d} x \mathrm{~d} y,
$$


where $\hat{\boldsymbol{F}}_{3 A}$ and $\hat{\boldsymbol{F}}_{3 A|A|^{2}}$ are the resonant forcing terms respectively proportional to the amplitudes $A$ and $A|A|^{2}$, whose expression is detailed in Appendix $\mathrm{B}$, and $\mathscr{S}$ is the scalar product between the direct and adjoint global modes

$$
\mathscr{S}=\int_{\Sigma} \hat{\boldsymbol{q}}_{1 A}^{\dagger} \cdot \hat{\boldsymbol{q}}_{1 A} \mathrm{~d} x \mathrm{~d} y .
$$

In (3.19), $\alpha_{x}$ is the receptivity to the cylinder $x$-displacement, i.e. the complex coefficient defined by

$$
\alpha_{x}=\mathscr{S}^{-1} \int_{\Upsilon_{w}}\left(\hat{p}_{1 A}^{\dagger} \boldsymbol{n}+\frac{1}{R e_{*}} \nabla \hat{\boldsymbol{u}}_{1 A}^{\dagger} \cdot \boldsymbol{n}\right) \cdot\left(\mathrm{i} \omega_{*} \boldsymbol{e}_{x}-\nabla \boldsymbol{u}_{0} \cdot \boldsymbol{e}_{x}\right) \mathrm{d} l,
$$

and $\alpha_{y}$ is the receptivity to the cylinder $y$-displacement, defined similarly by substituting $\boldsymbol{e}_{x}$ by $\boldsymbol{e}_{y}$ in (3.20). Finally, $\eta$ is the receptivity to the wall blowing and suction velocity, i.e. the complex coefficient defined by

$$
\eta=\mathscr{S}^{-1} \int_{\Upsilon_{w}}\left(\hat{p}_{1 A}^{\dagger} \boldsymbol{n}+\frac{1}{R e_{*}} \nabla \hat{\boldsymbol{u}}_{1 A}^{\dagger} \cdot \boldsymbol{n}\right) \cdot \boldsymbol{U}_{\boldsymbol{c}} \mathrm{d} l .
$$

It can be seen from (3.20)-(3.21) that the action of a resonant wall velocity on the global mode amplitude, which comes either from the retroaction of the cylinder displacement or from the externally applied control velocity, is determined by two distinct contributions associated with mass conservation and viscous effects: the first term, involving the adjoint pressure $\hat{p}_{1 A}^{\dagger}$ taken at the wall, accounts for the effect of the mass flux, whereas the second term, weighted by the inverse of the Reynolds number, takes into account the modification of the viscous friction at the wall when the cylinder is moving or when blowing and suction is applied.

\subsection{Structure model}

It can be checked that the linear oscillator equations (2.3) are straightforwardly satisfied at order $\epsilon^{3}$ with the present balance. As stated previously, the order $\epsilon^{3}$ cylinder displacement is forced near-resonance by order $\epsilon^{5}$ drag and lift forces, thus yielding the amplitude equations

$$
\frac{\mathrm{d} X}{\mathrm{~d} T}=\omega_{*}(-\Gamma+\mathrm{i} \Omega) X+\frac{\beta_{x}}{\omega_{*} M} A, \quad \frac{\mathrm{d} Y}{\mathrm{~d} T}=\omega_{*}(-\Gamma+\mathrm{i} \Omega) Y+\frac{\beta_{y}}{\omega_{*} M} A .
$$

In (3.22), $\beta_{x}$ is the complex drag coefficient defined by

$$
\beta_{x}=\frac{2 \mathrm{i}}{\pi} \int_{\Upsilon_{w}}\left(\hat{p}_{1 A} \boldsymbol{n}-\frac{1}{R \boldsymbol{e}_{*}}\left(\nabla \hat{\boldsymbol{u}}_{1 A}+\nabla \hat{\boldsymbol{u}}_{1 A}^{\mathrm{T}}\right) \cdot \boldsymbol{n}\right) \cdot \boldsymbol{e}_{\boldsymbol{x}} \mathrm{d} l,
$$

and $\beta_{y}$ its lift counterpart obtained by substituting $\boldsymbol{e}_{x}$ by $\boldsymbol{e}_{y}$ is (3.23).

\subsection{Coupled model}

Since the global mode $\hat{\boldsymbol{q}}_{1 \mathrm{~A}}$ is antisymmetric with respect to the $y=0$ axis, i.e. it satisfies $(u, v, p) \longrightarrow(-u, v,-p)$ under the $y \rightarrow-y$ reflection, it can be shown that $\alpha_{x}=\beta_{x}=0$, meaning that the linear oscillator equation in $X$ is decoupled from the flow motion. By choosing the phase of the initial displacement to be zero, its solution reads as

$$
\begin{aligned}
X(T) & =X(0) \exp \left[\omega_{*}(-\Gamma+\mathrm{i} \Omega) T\right], \\
\text { i.e. } \quad x_{s}(t) & =x_{s}^{0}+\left(x_{s}(0)-x_{s}^{0}\right) \mathrm{e}^{-\omega_{*} \gamma t} \cos \left(\omega_{s} t\right) .
\end{aligned}
$$


When $\gamma=0$, the streamwise displacement is therefore time-periodic and the cylinder oscillates with the natural frequency $\omega_{s}$. When $\gamma \neq 0$, the streamwise displacement is underdamped: the cylinder then oscillates at the same frequency but returns to its initial position with an exponential decay rate $\omega_{*} \gamma$. Consequently, we set $x_{s}=0$ and restrict in the following to a pure transverse displacement of the cylinder.

From now on, we consider only the uncontrolled case, for which $\eta=0$. In order to ease the notation, we also drop the subscript $y$ for $\alpha_{y}$ and $\beta_{y}$, so that the coupled equations read as

$$
\begin{aligned}
& \frac{\mathrm{d} A}{\mathrm{~d} T}=\lambda \delta A-\mu A|A|^{2}+\alpha Y, \\
& \frac{\mathrm{d} Y}{\mathrm{~d} T}=\omega_{*}(-\Gamma+\mathrm{i} \Omega) Y+\frac{\beta}{\omega_{*} M} A .
\end{aligned}
$$

System (3.25) is expressed in terms of the rescaled amplitudes $A$ and $Y$, but can be recast equivalently in terms of the physically meaningful amplitudes $\mathscr{A}=\epsilon A$ and $\mathscr{Y}=\epsilon^{3} Y$. The obtained system is similar and reads as

$$
\begin{aligned}
\frac{\mathrm{d} \mathscr{A}}{\mathrm{d} t} & =\lambda\left(\frac{1}{R e_{*}}-\frac{1}{R e}\right) \mathscr{A}-\mu \mathscr{A}|\mathscr{A}|^{2}+\alpha \mathscr{Y}, \\
\frac{\mathrm{d} \mathscr{Y}}{\mathrm{d} t} & =\left[-\omega_{*} \gamma+\mathrm{i}\left(\omega_{s}-\omega_{*}\right)\right] \mathscr{Y}+\frac{\beta}{\omega_{*} m} \mathscr{A} .
\end{aligned}
$$

System (3.25) is appropriate to identify the order in the expansion of each individual effect, as it depends only on rescaled quantities of order one. In contrast, system (3.26) is more convenient to discuss the physics, as it depends only on the physical variables, namely the fast time $t$, the Reynolds number and the structural parameters. In practice, all numerical results presented in the following issue from the resolution of (3.26), for which no value of the expansion parameter $\epsilon$ needs to be prescribed in the numerics.

\subsection{Computation of the model parameters}

The numerical approach retained to compute the coefficients $\lambda, \mu, \alpha, \beta$ and the various flow fields of interest is based on a finite element method and is similar to that used by Sipp \& Lebedev (2007) for a fixed cylinder. The computational domain used in the numerics is delimited by the cylinder wall $\Upsilon_{w}$ and the boundaries $\Upsilon_{i n}$ (inlet), $\Upsilon_{\text {out }}$ (outlet) and $\Upsilon_{\text {ext }}$ (external boundary), located respectively at $x=x_{-\infty}, x=x_{\infty}$ and $|y|=y_{\infty}$ with presently $x_{-\infty}=-100, x_{\infty}=150$ and $y_{\infty}=25$. We impose a uniform inlet condition $\boldsymbol{u}=(1,0)^{\mathrm{T}}$ on $\Upsilon_{i n}$ and no-stress conditions $\left(-p \boldsymbol{I}+R e^{-1} \nabla \boldsymbol{u}\right) \cdot \boldsymbol{n}=\mathbf{0}$ at the outlet $\Upsilon_{\text {out }}$. On the external boundary $\Upsilon_{\text {ext }}$, we impose a free-slip condition $\partial_{y} u=v=0$, so that the cylinder wall is the only source of vorticity, as would be the case without this artificial boundary.

The FreeFem++ software (http://www.freefem.org) is used to generate a mesh composed of triangular elements with the Delaunay-Voronoi algorithm. The mesh refinement is controlled by the vertex densities on both external and internal boundaries. The unknown velocity and pressure fields are spatially discretized using a basis of Taylor-Hood elements, i.e. P2 elements ( 6 degrees of freedom) for velocities and P1 elements ( 3 degrees of freedom) for pressure. The associated variational formulations are derived and spatially discretized on the mesh, and the sparse matrices resulting from the projection of the variational formulations onto the basis of finite 
elements are built with the FreeFem ++ software. The complete resolution procedure can be summarized as follows.

(a) The base flow $\boldsymbol{q}_{0}$ solution at order $\epsilon^{0}$ is obtained solving the nonlinear equations (3.12) for a fixed cylinder. To this end, we use an iterative Newton method (Barkley, Gomes \& Henderson 2002) involving the resolution of simple linear problems. The matrix inverses are then computed using the UMFPACK library, which consists in a sparse direct LU solver (Davis \& Duff 1997; Davis 2004; also http://www.cise.ufl.edu/research/sparse/umfpack).

(b) To obtain the solution at order $\epsilon^{1}$, i.e. the direct mode $\hat{\boldsymbol{q}}_{1 A}$, we solve the eigenvalue problem (3.2) along with homogeneous boundary conditions using the 'implicitly restarted Arnoldi method' of the ARPACK library (http://www.caam.rice.edu/software/ARPACK), based upon a shift and invert strategy (Ehrenstein \& Gallaire 2005). Here $\hat{\boldsymbol{q}}_{1 A}$ is normalized so as to obtain a coupling coefficient $\beta=1$, hence meaning that the phase of the fluctuating lift force is simply equal to the phase of the complex amplitude $A$.

(c) The solution at order $\epsilon^{2}$ is obtained by solving the linear problems (3.15) with the same boundary conditions as for the global mode, the matrix inversion being again carried out using the UMFPACK library.

(d) Finally, the adjoint global mode $\hat{\boldsymbol{q}}_{1 A}^{\dagger}$ needed to enforce the compatibility condition is obtained solving the adjoint eigenvalue problem (3.16) with the Arnoldi method already used for the direct mode. The adjoint boundary conditions to be satisfied are such that all boundary terms arising during the integration by parts are zero. We thus use the same boundary conditions as for the global mode, except at the outlet, where we impose the adjoint outflow condition $\left(\boldsymbol{u}_{0} \cdot \boldsymbol{n}\right) \hat{\boldsymbol{u}}_{1 A}^{\dagger}+\left(\hat{p}_{1 A}^{\dagger} \boldsymbol{I}+R e^{-1} \nabla \hat{\boldsymbol{u}}_{1 A}^{\dagger}\right) \cdot \boldsymbol{n}=\mathbf{0}$. The phase and amplitude of the adjoint global mode are then normalized so as to satisfy

$$
\mathscr{S}=\int_{\Sigma} \hat{\boldsymbol{q}}_{1 A}^{\dagger} \cdot \hat{\boldsymbol{q}}_{1 A} \mathrm{~d} x \mathrm{~d} y=1 .
$$

The flow fields appearing at each order of the asymptotic expansion are presented in figure 2. They exhibit a good agreement with those documented by Sipp \& Lebedev (2007, see figure 3 therein for comparison). The difference in the level of magnitude observed for the various solutions results from the different normalization of the global mode, as it can be seen from (3.15)-(3.27) that a change in $\hat{\boldsymbol{q}}_{1 \mathrm{~A}}$ by a factor $\Pi$ leaves the second-order solution $\hat{\boldsymbol{q}}_{2 \delta}$ unchanged, but yields a change in $\hat{\boldsymbol{q}}_{1 A}^{\dagger}, \hat{\boldsymbol{q}}_{2|A|^{2}}$ and $\hat{\boldsymbol{q}}_{2 A^{2}}$ by a factor $1 / \Pi,|\Pi|^{2}$ and $\Pi^{2}$, respectively. We find that the steady flow becomes unstable at the critical Reynolds number $R e_{*}=46.6$, the marginal frequency corresponding to a Strouhal number $S t_{*}=0.117$. Such results are consistent with those documented in the literature $\left(R e_{*}=46.7, S t_{*}=0.118\right.$ in Giannetti \& Luchini 2007 and $R e_{*}=46.6, S t_{*}=0.117$ in Sipp \& Lebedev 2007). Computing ultimately the different parameter values, we obtain

$\lambda=9.153+3.239 \mathrm{i}, \quad \mu=308.9-1025 \mathrm{i}, \quad \alpha=0.03492+0.01472 \mathrm{i}, \quad \beta=1$,

these values being converged down to the third digit, as evidenced in Appendix A. It can be shown that both the linear coefficient $\lambda$ and the ratio $\mu_{r} / \mu_{i}=-3.32$ are intrinsic quantities, i.e. they do not depend on the normalization of the global mode, the present results being in excellent agreement with those reported by Sipp \& Lebedev (2007), namely $\lambda=9.1+3.3 \mathrm{i}$ and $\mu_{i} / \mu_{r}=-3.4$. 
(a)

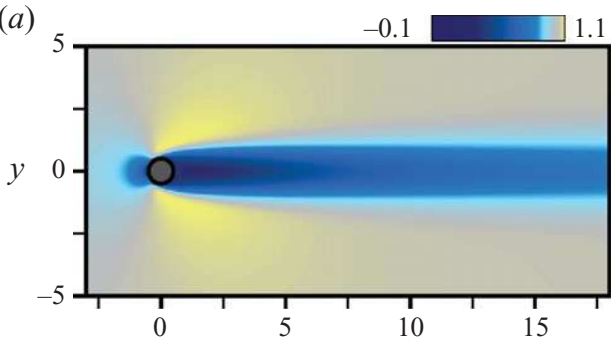

(c)

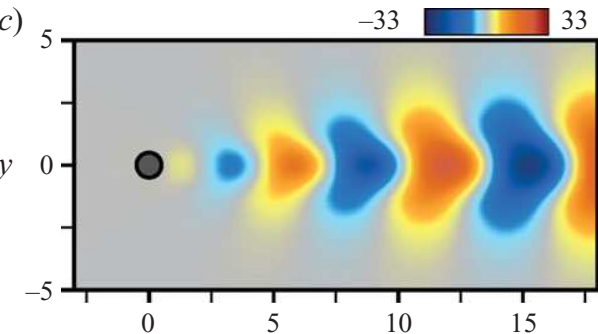

(e)

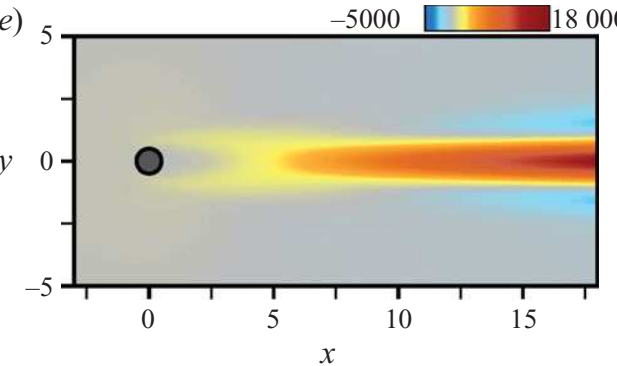

(b)

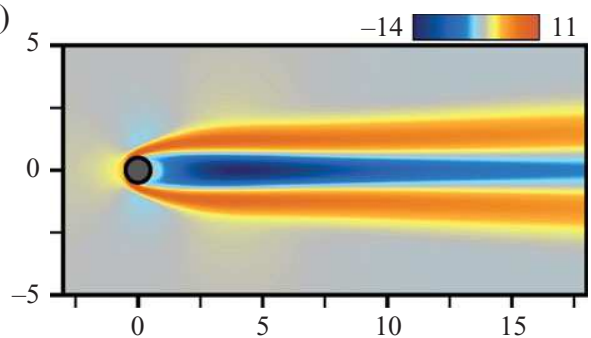

(d)



$(f)$

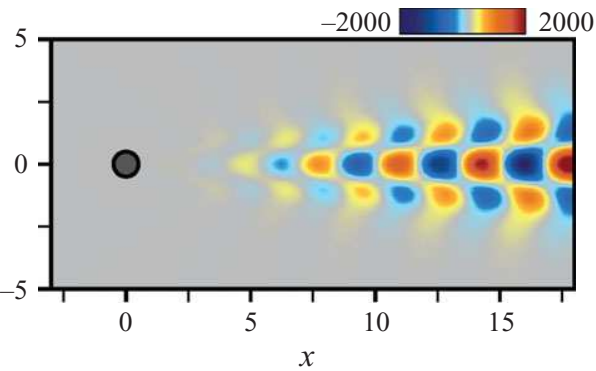

FIGURE 2. Representation of the various flow fields appearing at each order in the weakly nonlinear analysis: streamwise velocity components of the $(a)$ base flow $\boldsymbol{q}_{0},(b)$ second-order solution $\hat{\boldsymbol{q}}_{2 \delta}$ accounting for the modification of the base flow when the Reynolds number is varied, $(e)$ second-order solution $\hat{\boldsymbol{q}}_{2|A|^{2}}$ representing the difference between the mean flow and the base flow and $(f)$ second-order harmonic contribution $\hat{\boldsymbol{q}}_{2 A^{2}}$. Cross-stream velocity components of the $(c)$ global mode $\hat{\boldsymbol{q}}_{1 A}$ and $(d)$ adjoint global mode $\hat{\boldsymbol{q}}_{1 A}^{\dagger}$.

\section{Linear analysis}

We investigate here the effect of the fluid-structure coupling on the transition to linear instability. The linearization of system (3.25) yields

$$
\begin{aligned}
& \frac{\mathrm{d} A}{\mathrm{~d} T}=\lambda \delta A+\alpha Y, \\
& \frac{\mathrm{d} Y}{\mathrm{~d} T}=\omega_{*}(-\Gamma+\mathrm{i} \Omega) Y+\frac{\beta}{\omega_{*} M} A .
\end{aligned}
$$

We thus seek the eigenvalues $\xi$ of a $2 \times 2$ matrix

$$
\left(\begin{array}{cc}
\lambda \delta & \alpha \\
\frac{\beta}{\omega_{*} M} & \omega_{*}(-\Gamma+\mathrm{i} \Omega)
\end{array}\right),
$$

which yields

$$
2 \xi=\lambda \delta+\omega_{*}(-\Gamma+\mathrm{i} \Omega) \pm \sqrt{\left(\lambda \delta-\omega_{*}(-\Gamma+\mathrm{i} \Omega)\right)^{2}+4 \frac{\alpha \beta}{\omega_{*} M}} .
$$


When recasting these results in terms of the fast time $t$, the eigenvalues of the coupled system read as $\mathscr{X}=\mathrm{i} \omega_{*}+\epsilon^{2} \xi$, meaning that the marginal eigenvalue $\mathrm{i} \omega_{*}$ is modified at second-order, both in growth rate and frequency, under the action of the Reynolds number variation and the cylinder displacement. The system is thus stable when both eigenvalues have negative real parts, and we note $R e_{c}$ the critical Reynolds number of the coupled system for which the largest real part becomes zero.

In practice, the linear analysis is carried out in terms of the physical variables, for which (4.2) is recast into

$$
\left(\begin{array}{cc}
\lambda\left(\frac{1}{R e_{*}}-\frac{1}{R e}\right) & \alpha \\
\frac{\beta}{\omega_{*} m} & -\omega_{*} \gamma+\mathrm{i}\left(\omega_{s}-\omega_{*}\right)
\end{array}\right)
$$

the eigenvalues being then

$$
\begin{aligned}
2 \mathscr{X}= & \lambda\left(\frac{1}{R e_{*}}-\frac{1}{R e}\right)-\omega_{*} \gamma+\mathrm{i}\left(\omega_{s}+\omega_{*}\right) \\
& \pm \sqrt{\left[\lambda\left(\frac{1}{R e_{*}}-\frac{1}{R e}\right)+\omega_{*} \gamma-\mathrm{i}\left(\omega_{s}-\omega_{*}\right)\right]^{2}+4 \frac{\alpha \beta}{\omega_{*} m}} .
\end{aligned}
$$

It can be shown from (4.5) that for each couple $\left(\gamma, \omega_{s}\right)$, the system admits a single double root only for a unique parameter setting denoted by $\left(R e_{0}, m_{0}\right)$. Provided the Reynolds number is sufficiently different from $R e_{0}$, it is therefore possible to follow the eigenvalues continuously up to large mass ratios. In this case, we recover the modes identified by Cossu \& Morino (2000) by defining the wake mode and the structure mode, such that the associated eigenvalues satisfy

$$
\begin{aligned}
\lim _{m \rightarrow \infty} \mathscr{X}=\mathrm{i} \omega_{*}+\lambda\left(\frac{1}{R e_{*}}-\frac{1}{R e}\right) & \text { (wake mode), } \\
\lim _{m \rightarrow \infty} \mathscr{X}=-\omega_{*} \gamma+\mathrm{i} \omega_{s} & \text { (structure mode). }
\end{aligned}
$$

Indeed, for large mass ratios, the flow-induced lift force becomes very small compared with the structural ones and the fluid-structure interaction drops to negligible levels, so that the eigenvalues are deduced straightforwardly from the diagonal terms of (4.4), with $\mathrm{i} \omega_{*}+\lambda\left(1 / R e_{*}-1 / R e\right)$ being the eigenvalue classically computed from the flow past a fixed cylinder, and $-\omega_{*} \gamma+\mathrm{i} \omega_{s}$ being the natural eigenvalue of the cylinder-only system.

We focus here on the effect of the mass ratio, keeping the structural damping and natural frequency constant and equal to $\gamma=0.01, \omega_{s}=0.75$. Figure $3(a)$ presents the critical Reynolds number $R e_{c}$ computed as a function of $m$. The flow is unstable for combinations of parameters located in the shaded region labelled $U$, and stable otherwise. The circle denotes the location of the Reynolds and mass ratios for which the eigenvalue coalescence occurs, namely $m_{0}=1274$ and $R e_{0}=45.5$. For $m \gtrsim 5000$, the threshold value is asymptotic to $R e_{c}=R e_{*}$, indicating that the mass ratio has little influence on the stability of the system, as a result of the limited fluid-structure interaction expected for such heavy cylinders. In contrast, for $m \lesssim 1000$, the critical Reynolds number drops dramatically as the mass ratio decreases. For instance, for $m=100$, we obtain $R e_{c}=35.5$, which represents a decrease by $\sim 25 \%$. Still, it should be kept in mind that the present results are only qualitative, as the asymptotic 

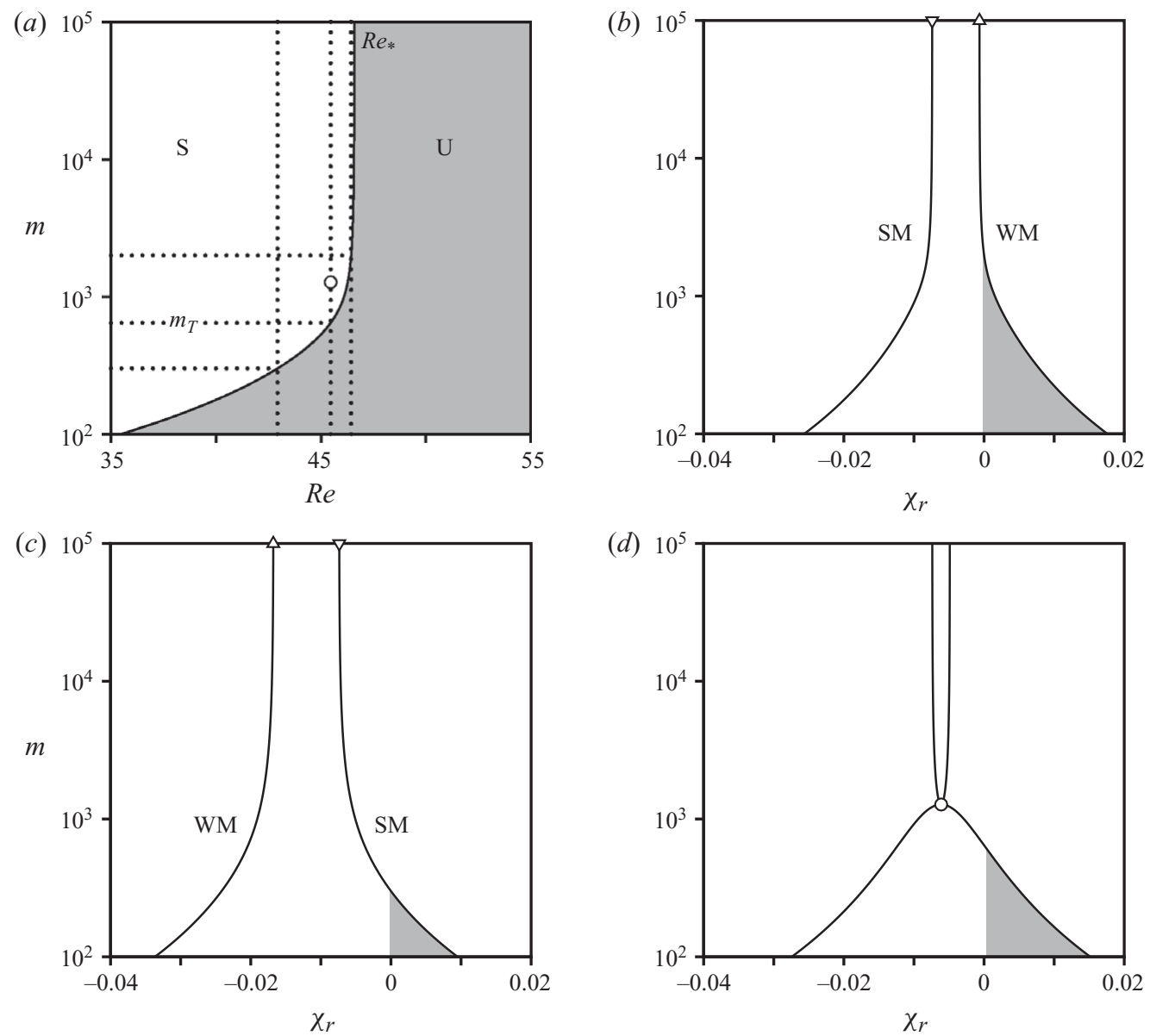

FIGURE 3. (a) Boundary separating the unstable domain (shaded area labelled U) from the stable domain (area labelled S) in the plane $(R e, m)$, for $\gamma=0.01$ and $\omega_{s}=0.75$. The circle indicates the setting $\left(R_{0}, m_{0}\right)$ for which the eigenvalue coalescence occurs. $(b)$ Growth rate of the wake mode (WM) and the structure mode (SM) as a function of the mass ratio, for $R e=46.4$ (rightmost vertical dotted line in $a$ ). (c) Same as $(b)$ for $R e=42.9$ (leftmost vertical dotted line in $a$ ). $(d)$ Same as $(b)$ for $R e_{0}$ at which the eigenvalue coalescence occurs (middle vertical dotted line in $a$ ).

expansion is meant for large mass ratios. As a comparison, Cossu \& Morino (2000) reported that a mass ratio $m=70$ yields a decrease of the critical Reynolds by more than $50 \%$, even though the forcing frequency is almost twice as large as the natural frequency.

For a given mass ratio $m$, it is now possible to determine whether the instability is led by the wake or the structure mode. Consider for instance the ratio $m=2000$ corresponding to the upper horizontal dotted line in figure 3( $a)$. In order to identify the leading mode, we set the critical Reynolds number to $\operatorname{Re}_{c}(2000) \sim 46.4$ and follow the eigenvalues continuously up to large mass ratios, hence moving along the rightmost vertical dotted line in figure $3(a)$. The growth rates obtained by doing so are presented in figure $3(b)$, where the WM and SM labels refer to the wake and structure modes, respectively. We retrieve that the coupled system is unstable for $m \leqslant 2000$ (grey shaded area) and stable otherwise. The asymptotic values expected in 


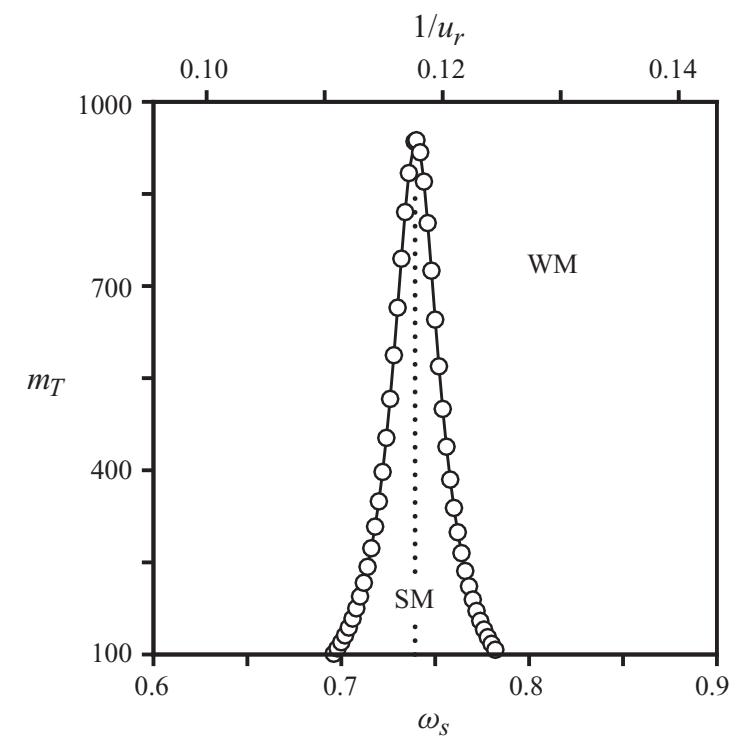

FIGURE 4. Locus of the cutoff ratio $m_{T}$ in the plane $\left(\omega_{s}, m\right)$. This curve separates domains where the transition to linear instability is led, respectively, by the structure mode (area labelled $\mathrm{SM}$ ) and the wake mode (area labelled WM).

the absence of coupling, are indicated by the symbols on the upper horizontal axis, and show that the instability is led by the wake mode (triangle symbol), whereas the structure mode (delta symbol) remains stable. Consider now the ratio $m=300$ corresponding to the lower horizontal dotted line in figure 3(a). Figure 3(c) presents the growth rates obtained by repeating the above procedure, the Reynolds number being set to $\operatorname{Re}_{c}(300) \sim 42.9$. It can be seen that the instability is now led by the structure mode, whereas the wake mode remains stable. The transition between these two regimes occurs for the mass ratio $m_{T}$, defined by $\operatorname{Re}_{c}\left(m_{T}\right)=R e_{0}\left(m_{T}=645\right.$ for the present parameter setting), for which results are shown in figure $3(d)$. In this case, no distinction can be made between a wake and a structure mode, since both branches exchange roles at $m=m_{0}$, where the eigenvalue coalescence occurs. Results may thus be summarized as follows.

(i) If $m \gtrsim m_{T}$, the instability is led by the wake mode.

(ii) If $m \lesssim m_{T}$, the instability is led by the structure mode.

(iii) If $m \sim m_{T}$, no clear distinction can be made between a wake and a structure mode. Detailed calculations show that the identification remains intricate if the Reynolds number departs from $R e_{0}$ by less than $0.5 \%$, a case for which the instability can be seen as being led by mixed wake/structure modes.

A cutoff ratio $m_{T}$ can be computed similarly for each value of the couple $\left(\gamma, \omega_{s}\right)$. The results obtained by varying the natural frequency and keeping the structural damping constant and equal to $\gamma=0.01$ are synthesized in figure 4 . The circles denote computational points, whereas the connecting line has been obtained by a spline interpolation. We find that the instability is led by the structure mode up to $m \sim 950$ for structural frequencies close to the marginal frequency $\omega_{*}$ (indicated by the vertical dotted line). In contrast, the instability is essentially led by the wake mode when $\omega_{s}$ departs from $\omega_{*}$, a parameter range for which the cutoff ratio drops dramatically. 

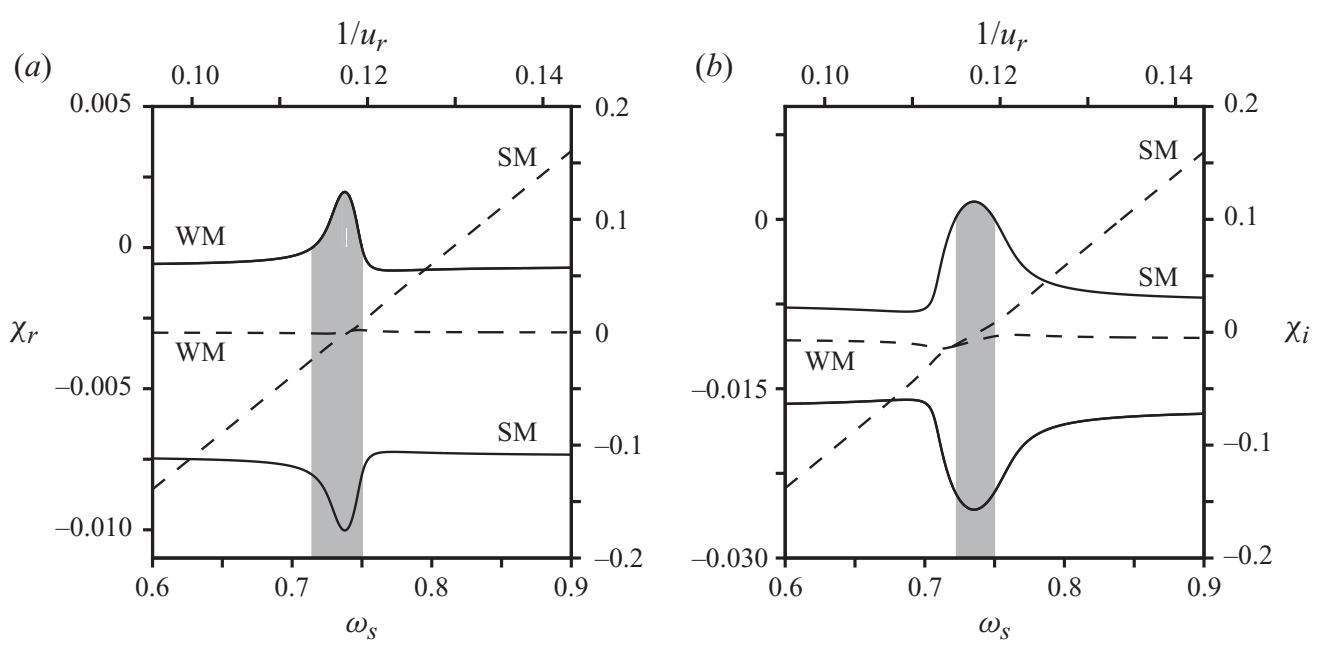

FIGURE 5. Effect of the natural frequency $\omega_{s}$ on the growth rate (solid lines) and frequency (dashed lines) of the wake and structure modes, for $\gamma=0.01$. The range of instability is indicated by the grey shaded area. (a) $m=2000$ and $R e=46.4$ : the transition is led by the wake mode. $(b) m=300$ and $R e=42.9$ : the transition is led by the structure mode.

We present in figure 5(a) the growth rates and frequencies of the wake and structure modes computed as functions of $\omega_{s}$ for $m=2000$ at $R e=46.4$. Different scales are used on the horizontal axis, which are labelled in terms of the frequency $\omega_{s}$ and the reduced velocity $u_{r}$. Consistent with the results presented in figure 3 , the structure mode remains stable and the instability is sustained by the wake mode (as indicated by the grey shaded area), in a range where the frequencies of both modes synchronize. Similar results are observed in figure $5(b)$, where we present the eigenvalues of both modes computed for $m=300$ at $R e=42.9$. The wake mode remains stable and the instability is now sustained by the structure mode. Moreover, it can be seen that the frequencies of both modes depart from their unforced values close to synchronization, but that the maximum of instability is not reached at synchronization in the most general case. Such results are reminiscent of those documented by de Langre (2006) using the wake oscillator model, for which linear instability results from coupled-mode flutter.

Although the linear analysis does not allow to provide quantitative predictions of the cylinder motion, the present results give credence to the interpretation of VIV in terms of a linear instability causing the fluctuating amplitude of the cylinder displacement to grow and ultimately saturate at a high but finite amplitude, under the action of nonlinearities indeed described below.

\section{Nonlinear analysis and limit cycles}

We now study the nonlinear dynamics of the coupled system (3.25) by setting $A=|A| \mathrm{e}^{\mathrm{i} \phi_{A}}$ and $Y=|Y| \mathrm{e}^{\mathrm{i} \phi_{Y}}$. In the following, we use the phase $\phi=\phi_{A}-\phi_{Y}$, representing the phase shift between the lift fluctuations induced by the vortex shedding and the cylinder displacement, since the normalization of the fluid eigenmode imposes the phase of the $\beta$-coefficient to be zero. Positive (respectively negative) values of $\phi$ mean that the cylinder is in phase delay (respectively phase advance) with respect to the lift fluctuations. This allows to recast (3.25) into a three-dimensional 
polar system for $|A|,|Y|$ and $\phi$ as

$$
\begin{aligned}
\frac{\mathrm{d}|A|}{\mathrm{d} T} & =\lambda_{r} \delta|A|-\mu_{r}|A|^{3}+|\alpha| \cos \left(\phi-\phi_{\alpha}\right)|Y|, \\
\frac{\mathrm{d}|Y|}{\mathrm{d} T} & =-\omega_{*} \Gamma|Y|+\frac{|\beta|}{\omega_{*} M} \cos \phi|A|, \\
\left(1+\tan ^{2} \phi\right) \frac{\mathrm{d} \phi}{\mathrm{d} T} & =\mathscr{F}_{3} \tan ^{3} \phi+\mathscr{F}_{2} \tan ^{2} \phi+\mathscr{F}_{1} \tan \phi+\mathscr{F}_{0},
\end{aligned}
$$

with $\mathscr{F}_{m}(m=0, \ldots, 3)$ being the real coefficients reading

$$
\left.\begin{array}{ll}
\mathscr{F}_{3}=-\omega_{*} \Gamma, & \mathscr{F}_{1}=\mathscr{F}_{3}-\frac{|\alpha| \beta}{\omega_{*}^{2} \Gamma M}\left(\cos \phi_{\alpha}+\frac{\mu_{i}}{\mu_{r}} \sin \phi_{\alpha}\right), \\
\mathscr{F}_{2}=\left(\lambda_{i}-\frac{\mu_{i}}{\mu_{r}} \lambda_{r}\right) \delta-\omega_{*} \Omega, & \mathscr{F}_{0}=\mathscr{F}_{2}+\frac{|\alpha| \beta}{\omega_{*}^{2} \Gamma M}\left(\sin \phi_{\alpha}-\frac{\mu_{i}}{\mu_{r}} \cos \phi_{\alpha}\right),
\end{array}\right\}
$$

and $\phi_{\alpha}=\arg (\alpha)=0.399\left(22.9^{\circ}\right)$ with the present normalization.

The analysis is first carried out in terms of the rescaled variables, so as to establish the order in the expansion of the quantities of interest. We restrict on to solutions characterized by a constant phase shift, i.e. such that $\mathrm{d} \phi / \mathrm{d} T=0$. In this case, $(5.1 c)$ defines $\phi$ as the solution of a third-order polynomial equation in $\tan \phi$, which yields either one or three real roots (note that $\phi$ is unique, as it comes from $(5.1 b)$ that $\phi \in[-\pi / 2 ; \pi / 2]$ on the limit cycle). To each value of $\phi$ corresponds a solution on the limit cycle, and it will be shown in $\S 5.2$ that hysteretic behaviours result from the existence of multiple roots, i.e. from the simultaneous existence of several limit cycles. On a given cycle, the amplitude $|A|$ and $|Y|$ solutions of $(5.1 a, b)$ are obtained as

$$
\begin{aligned}
& |A|=\sqrt{\frac{\lambda_{r}}{\mu_{r}} \delta+\frac{|\alpha| \beta}{\mu_{r} \omega_{*}^{2} \Gamma M} \cos \left(\phi-\phi_{\alpha}\right) \cos \phi}, \\
& |Y|=\frac{\beta}{\omega_{*}^{2} \Gamma M} \cos \phi|A| .
\end{aligned}
$$

Since system (3.25) is to be satisfied, the individual phases $\phi_{A}$ and $\phi_{Y}$ are eventually recovered as

$$
\phi_{A}(T)=\Omega_{A} T, \quad \phi_{Y}(T)=\Omega_{Y} T-\phi(0),
$$

where we have imposed the phase of the flow initial disturbance to be zero, and $\Omega_{A}$ and $\Omega_{Y}$ are detuning parameters defined by

$$
\begin{aligned}
& \Omega_{A}=\left(\lambda_{i}-\frac{\mu_{i}}{\mu_{r}} \lambda_{r}\right) \delta-\frac{|\alpha| \beta}{\omega_{*}^{2} \Gamma M} \cos \phi\left(\sin \left(\phi-\phi_{\alpha}\right)+\frac{\mu_{i}}{\mu_{r}} \cos \left(\phi-\phi_{\alpha}\right)\right), \\
& \Omega_{Y}=\omega_{*} \Omega+\omega_{*} \Gamma \tan \phi .
\end{aligned}
$$

Note that the phase $\phi$ is constant at all times for the present limit-cycle solutions. This imposes $\Omega_{A}=\Omega_{Y}$, which is equivalent to $(5.1 c)$.

When recasting these results in terms of the fast time $t$, the vortex-shedding frequency on the limit cycle reads as $\omega_{v}=\omega_{*}+\epsilon^{2} \Omega_{A}$. The detuning $\Omega_{A}$ therefore acts as a second-order frequency shift stemming from three distinct origins. The first term on the right-hand side of $(5.5 a)$ is due to linear mechanisms, namely the variation of the global eigenmode frequency as the Reynolds number varies, the second term is due to the nonlinear mechanisms, whereas the third term arises from the feedback exerted by the displacement of the structure. If we define $\omega_{v, f}$ as the saturated 
vortex-shedding frequency for a fixed cylinder, i.e.

$$
\omega_{v, f}=\omega_{*}+\epsilon^{2}\left(\lambda_{i}-\frac{\mu_{i}}{\mu_{r}} \lambda_{r}\right) \delta
$$

the vortex-shedding frequency of the coupled system can be rewritten as

$$
\omega_{v}=\omega_{v, f}-\epsilon^{2} \frac{|\alpha| \beta}{\omega_{*}^{2} \Gamma M} \cos \phi\left(\sin \left(\phi-\phi_{\alpha}\right)+\frac{\mu_{i}}{\mu_{r}} \cos \left(\phi-\phi_{\alpha}\right)\right) .
$$

Similarly, the cylinder frequency on the limit cycle can be written as $\omega_{c y l}=\omega_{*}+\epsilon^{2} \Omega_{Y}$. It can also be rewritten as a function of the natural frequency $\omega_{s}=\omega_{*}\left(1+\epsilon^{2} \Omega\right)$, according to

$$
\omega_{c y l}=\omega_{s}+\epsilon^{2} \omega_{*} \Gamma \tan \phi .
$$

In practice, the nonlinear analysis is carried out in terms of the physical variables, which requires to derive first the equivalent form of system (5.1), as has been done in $\S \S 3.3$ and 3.4. This step is straightforward and the analytical expression is voluntarily omitted for conciseness, but it is worthwhile noting that the value of $\phi$ is identical in both forms. Relation (5.3) can now be rewritten as

$$
\begin{aligned}
|\mathscr{A}| & =\sqrt{\frac{\lambda_{r}}{\mu_{r}}\left(\frac{1}{R e_{*}}-\frac{1}{R e}\right)+\frac{|\alpha| \beta}{\mu_{r} \omega_{*}{ }^{2} \gamma m} \cos \left(\phi-\phi_{\alpha}\right) \cos \phi,} \\
|\mathscr{Y}| & =\frac{\beta}{\omega_{*}{ }^{2} \gamma m} \cos \phi|\mathscr{A}| .
\end{aligned}
$$

Similarly, the vortex-shedding and cylinder frequencies can be recast into

$$
\begin{aligned}
\omega_{v, f} & =\omega_{*}+\left(\lambda_{i}-\frac{\mu_{i}}{\mu_{r}} \lambda_{r}\right)\left(\frac{1}{R e_{*}}-\frac{1}{R e}\right), \\
\omega_{v} & =\omega_{v, f}-\frac{|\alpha| \beta}{\omega_{*}^{2} \gamma m} \cos \phi\left(\sin \left(\phi-\phi_{\alpha}\right)+\frac{\mu_{i}}{\mu_{r}} \cos \left(\phi-\phi_{\alpha}\right)\right), \\
\omega_{c y l} & =\omega_{s}+\omega_{*} \gamma \tan \phi .
\end{aligned}
$$

The coupled solutions being synchronized, the limit-cycle frequencies are such that

$$
\omega_{v}=\omega_{c y l} .
$$

\subsection{Effect of the natural frequency}

We set here $\gamma=0.01$ and $m=10000$ and investigate the effect of the natural frequency on the cylinder motion. We present in figure 6(a) the evolution of the vortex-shedding frequency $\omega_{v}$ at $R e=60$. The dashed line is the bisector of the plane $\left(\omega_{v}, \omega_{s}\right)$, where the vortex-shedding frequency would be equal to the natural frequency of the structure. The horizontal dotted line represents the vortex-shedding frequency $\omega_{v, f}$ of the fixed cylinder, which departs from $\omega_{*}$ since the Reynolds number is supercritical. When the natural frequency is close to $\omega_{v, f}$, the vortex-shedding frequency departs from its unforced value and synchronizes onto the forcing frequency. The synchronization happens when both curves cross, which occurs for a specific value denoted by $\omega_{s}^{\max }$ (middle vertical dotted line), hence defining a lock-in state. The solid line in figure $6(b)$ depicts the evolution of the cylinder displacement amplitude $|\mathscr{Y}|$, computed as a function of $\omega_{s}$ for the same Reynolds number (solid line): we find large displacement amplitudes in the lock-in regime, as $|\mathscr{Y}|$ reaches a maximum precisely for $\omega_{s}=\omega_{s}^{\max }$. 

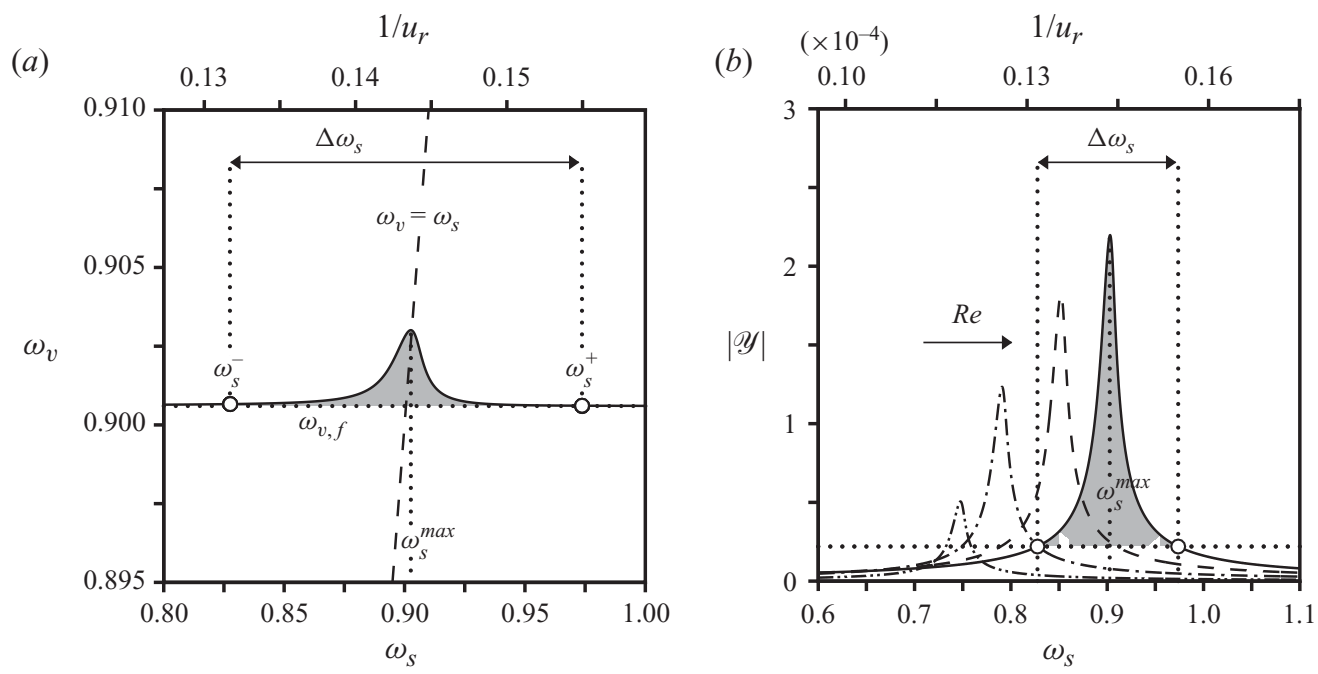

FIGURE 6. Effect of the natural frequency $\omega_{s}$ for $\gamma=0.01$ and $m=10000$. (a) Vortex-shedding frequency $\omega_{v}$ as a function of $\omega_{s}$ for $R e=60$. The horizontal dotted line indicates the value of the natural vortex-shedding frequency $\omega_{v, f}$ in the wake of a fixed cylinder, whereas the dashed line represents the bisector of the plane $\left(\omega_{v}, \omega_{s}\right)$. (b) Amplitude of the cylinder displacement $|\mathscr{Y}|$ as a function of $\omega_{s}$ for $R e=47$ (dash-dot-dotted line), $R e=50$ (dash-dotted line), $R e=55$ (dashed line) and $R e=60$ (solid line). For both plots, the grey shaded areas correspond to the resonance width $\Delta \omega_{s}=\omega_{s}^{+}-\omega_{s}^{-}$, for which the displacement exceeds $10 \%$ of its maximal amplitude, whose lower and upper bounds are indicated by the circles.

In contrast, the structure is almost at rest at lock-out. Similar results are obtained for $R e=55$ (dashed line), $R e=50$ (dash-dotted line) and $R e=47$ (dash-dot-dotted line), the maximum amplitude of the cylinder displacement increasing monotonically with the Reynolds number. A similar magnification is found for the amplitude of the global mode $|\mathscr{A}|$ (not shown here). The frequency shift $\omega_{v}-\omega_{v, f}$ observed in figure 6(a) at lock-in may therefore be viewed as a nonlinear shift induced by this increase in the vortex-shedding activity.

As for forced oscillators, we define a resonance width $\Delta \omega_{s}=\omega_{s}^{+}-\omega_{s}^{-}$within which the cylinder displacement amplitude is larger than a particular fraction of the amplitude at resonance (chosen here as $10 \%$ ), which allows a quantitative description of the lock-in state. The resonance width is shown for $R e=60$ by the grey shaded domain in figure 6 , the position of $\omega_{s}^{+}$and $\omega_{s}^{-}$being indicated by the open circles. Note that $\Delta \omega_{s}$ has also been computed as a function of the Reynolds number. The resulting lock-in domain in the plane $\left(\omega_{s}-R e\right)$ is indicated by the grey shade in figure $7(a)$. We find that lock-in occurs for $R e \gtrsim 46.4$. The resonance width then increases monotonically with the Reynolds number and rapidly reaches an almost constant value of $\Delta \omega_{s} \sim 0.15$.

We also show in figure 7(b) the evolution of the phase shift $\phi$ computed as a function of $\omega_{s}$ for the same Reynolds numbers as in figure $6(b)$. We observe systematically that $\phi\left(\omega_{s}^{\max }\right) \sim 0$, as indicated by the circles, meaning that the cylinder displacement is in phase with respect to the lift fluctuations at lock-in. When the natural frequency is smaller than the nonlinear vortex-shedding frequency, the cylinder is in retarded quadrature with respect to the lift fluctuations, i.e. $\phi \sim+\pi / 2$. When the natural 
(a)

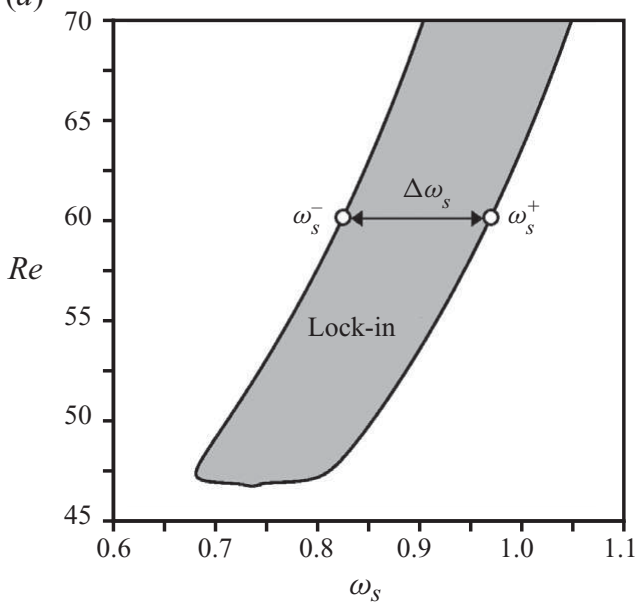

(b)

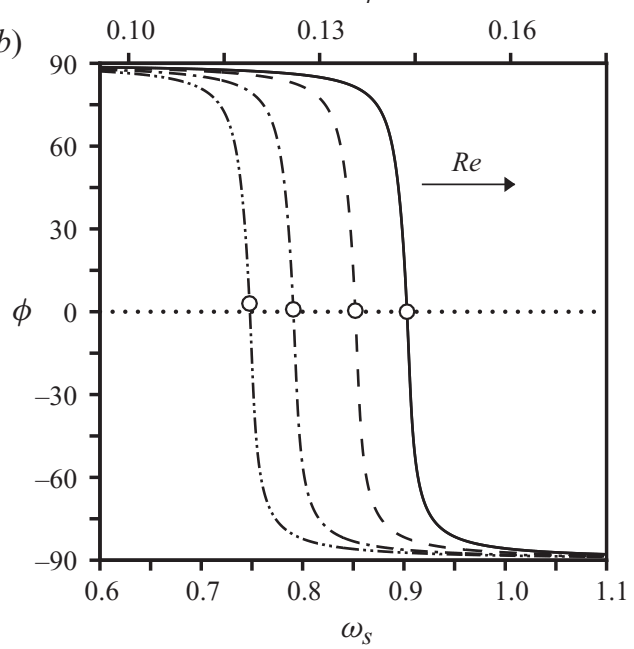

FiguRE 7. Effect of the natural frequency $\omega_{s}$ for $\gamma=0.01$ and $m=10000$. (a) Boundary of the resonance width $\Delta \omega_{s}$ as a function of the Reynolds number. The inner grey shade therefore defines the lock-in state. $(b)$ Phase shift $\phi$ as a function of $\omega_{s}$ for the same Reynolds numbers as in figure $6(b)$. The circles denote the value $\phi\left(\omega_{s}^{\max }\right)$ at lock-in.

frequency is larger than the vortex-shedding frequency, the cylinder is in advanced quadrature, i.e. $\phi \sim-\pi / 2$.

\subsection{Effect of the mass ratio}

We now consider the effect of the mass ratio on the cylinder motion, the structural damping being $\gamma=0.01$. Figure $8(a)$ shows the evolution of the vortex-shedding frequency for $m=100$ and $R e=50$. As in figure $6(a)$, the dashed line is the bisector of the plane $\left(\omega_{v}, \omega_{s}\right)$ and the horizontal dotted line is the vortex-shedding frequency $\omega_{v, f}$ of the fixed cylinder. Results are somehow similar to those reported in $\S 5.1$, although we observe the onset of a hysteretic behaviour corresponding to the transition from a one-real-root to a three-real-roots regime when solving for the phase shift equation recast from $(5.1 c)$. As a consequence, the vortex-shedding frequency rapidly departs from its unforced value and remains quasi-synchronized onto the forcing over a large range of frequencies. Still, it is possible to define a lock-in state for the frequency $\omega_{s}^{\max }$ at which the exact synchronization occurs. We have checked that the phase shift behaviour, although distorted by the simultaneous existence of distinct limit cycles, is also similar, namely the cylinder displacement is in phase with respect to the lift fluctuations at lock-in, and in quadrature at lock-out (not shown here for conciseness).

We also present in figure $8(b)$ the evolution of the normalized displacement amplitude computed as a function of $\omega_{s}$ for the same parameter setting (solid line) and find that $|\mathscr{Y}|$ again reaches its maximum value precisely at the lockin frequency $\omega_{s}^{\max }$. Similar results are obtained for mass ratios $m=200$ (dashed line), $m=500$ (dash-dotted line) and $m=1000$ (dash-dot-dotted line), all distributions having been normalized by their maximum value to ease the comparison. Interestingly, the hysteretic behaviour, which occurs at mass ratios $m \lesssim 2000$ for the Reynolds number and the damping parameter considered here, yields a monotonic increase of the lock-in frequency. It also yields a significant increase of the resonance width, whose upper bound is triggered by the tail-end of the hysteresis loop. For instance, we 
(a)

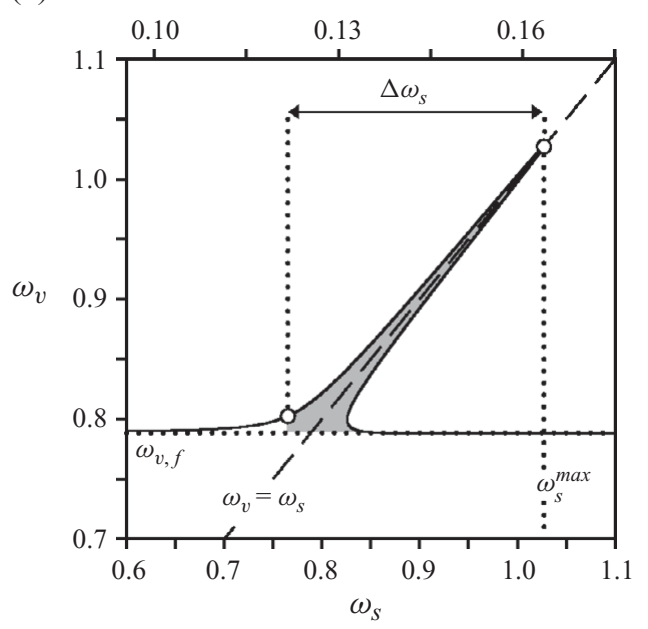

(b)

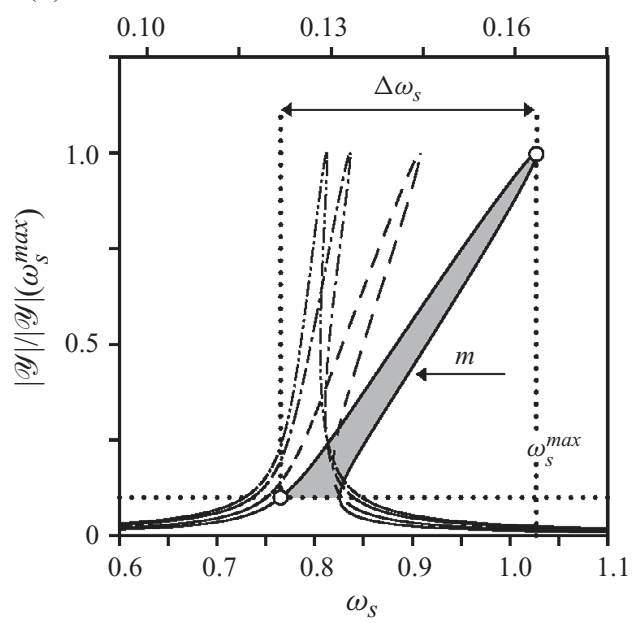

FiguRE 8. Effect of the mass ratio for $\gamma=0.01$ and $R e=50$. (a) Vortex-shedding frequency $\omega_{v}$ as a function of $\omega_{s}$ for $m=100$. The horizontal dotted line indicates the value of the natural vortex-shedding frequency $\omega_{v, f}$ in the wake of a fixed cylinder, whereas the dashed line is the bisector of the plane $\left(\omega_{v}, \omega_{s}\right)$. $(b)$ Amplitude of the cylinder displacement $|\mathscr{Y}|$ as a function of $\omega_{s}$, for $m=1000$ (dash-dot-dotted line), $m=500$ (dash-dotted line), $m=200$ (dashed line) and $m=100$ (solid line). The amplitude on each curve has been normalized by its maximum value.

find $\Delta \omega_{s} \sim 0.1$ for $m=500$ but $\Delta \omega_{s} \sim 0.26$ for $m=100$, such results being consistent with the generally acknowledged idea that the range of frequencies over which largeamplitude vibrations can be observed increases at low mass ratios, as early reported by Ramberg \& Griffin (1981).

\section{Extension of the model to small mass ratios and mass-damping parameters}

When used along with low mass ratios, the model presented in $\S 3$ yields somewhat surprising results: for instance, there is no lower threshold to how much the magnitude of the coupling can decrease the critical Reynolds numbers issuing from the linear analysis. Similarly, there is no upper threshold to how much it can increase the cylinder displacement amplitude predicted by the nonlinear analysis. The same remark holds for vanishing structural damping, as one sees from $(5.3 b)$ that a finite damping is required for the limit-cycle solution to saturate at a finite amplitude, a result classical of oscillators forced near resonance (Landau \& Lifshitz 1976).

Such results are not consistent with experimental observations, where one generally expects the structure to exhibit an asymptotic, self-limiting response. This can be explained simply by recalling that the structure equation has been obtained by setting the inertial and lift forces in balance at order $\epsilon^{5}$. In particular, it can be seen from (3.23) that the force exerted on the cylinder is that induced by the bifurcating global mode, as would be the case if the cylinder were fixed. The model therefore fails to capture two effects of practical importance for a body moving in a surrounding fluid.

(i) An accelerating or decelerating body displaces a certain mass of fluid responsible for an added-mass force opposing the motion (Govardhan \& Williamson 2000).

(ii) Owing to the body displacement, the relative free-stream velocity varies, which induces a change of the exerted drag force responsible for an added damping force (Blevins 1990). 
Both effects arise naturally for a solution to the Navier-Stokes equations. In the present case, they arise from the flow response to the cylinder displacement which comes at order $\epsilon^{3}$ in the asymptotic development. Since the mass ratio is of order $\epsilon^{-4}$, the corresponding forces come at order $\epsilon^{7}$ and can be legitimately neglected when compared with the retained lift force of order $\epsilon^{5}$. The original model is thus exact in the range of mass ratios for which it has been derived rigorously.

We propose to derive an extended model relying on the same structure equation at order $\epsilon^{5}$, now complemented by smaller terms issuing from the added-mass and added damping forces. These terms are not computed from the exact flow responses arising at successive orders, which would require intricate computations lying out of the scope of the present study. As is classically done in the literature, their contribution to the total lift force is accounted for by separate terms introduced by hand in the linear oscillator equation for $y_{s}$, which now reads as

$$
\frac{\mathrm{d}^{2} y_{s}}{\mathrm{~d} t^{2}}+2 \omega_{s} \gamma \frac{\mathrm{d} y_{s}}{\mathrm{~d} t}+\omega_{s}^{2} y_{s}=\frac{2}{\pi m} c_{y}-\frac{c_{a}}{m} \frac{\mathrm{d}^{2} y_{s}}{\mathrm{~d} t^{2}}-\frac{4}{\pi m} \omega_{s} \zeta \frac{\mathrm{d} y_{s}}{\mathrm{~d} t} .
$$

In (6.1), $c_{a}$ is the added-mass coefficient, whose value depends only on the geometry of the structure, the value usually reported for a circular cross-section being $c_{a}=1$. Likewise, $\zeta$ is the added damping coefficient, also referred as the stall parameter (Skop \& Balasubramanian 1997) and is defined from the mean drag coefficient of the structure as $\zeta=c_{x}^{0} / 2 \omega_{s}$ (Blevins 1990).

The system (3.25) remains invariant provided the structural parameters are transformed according to

$$
\begin{aligned}
\gamma & \rightarrow \frac{m}{m+c_{a}} \frac{\omega_{s}}{\omega_{*}}\left(\gamma+\frac{2}{\pi m} \zeta\right), \\
\omega_{s} & \rightarrow \sqrt{\frac{m}{m+c_{a}}} \omega_{s}, \\
m & \rightarrow m+c_{a} .
\end{aligned}
$$

Note that the so-called mass-damping parameter $\gamma m$ arises naturally on the righthand side of $(6.2 a)$. It can be checked that both sets of parameters are identical at leading-order when using the balance defined in $\S 3$, a limit in which the original model is retrieved. Only in the limit of low mass ratios and low mass-damping do the corrective terms acquire significance. In practice, they put an upper bound on the effective mass and damping terms, which is believed to trigger the expected self-limiting structural behaviour.

As an illustration, we present in figure $9(a)$ the critical Reynolds number, $R e_{c}$, computed as a function of $m$ with the extended model, the flow being unstable for combinations of parameters located in the shaded region labelled $U$, and stable otherwise. The results are reminiscent of those provided in figure $3(a)$, as the critical Reynolds numbers predicted by the exact and the extended models differ by less than $1 \%$ for mass ratios $m>450$, and by less than $5 \%$ for $m>150$. Still, the extended model now predicts the existence of a threshold value $R e \sim 32$, below which the coupling cannot decrease the critical Reynolds number, no matter the value of the mass ratio.

We now propose to further investigate the effect of the mass ratio on the lock-in domain. Figure $9(b)$ presents the resonance width computed as a function of $m$ using the extended model. The results obtained using the original model are shown by the dashed line in the range $m>100$, both sets differing by less than $2 \%$ for $m>450$ and 
(a)

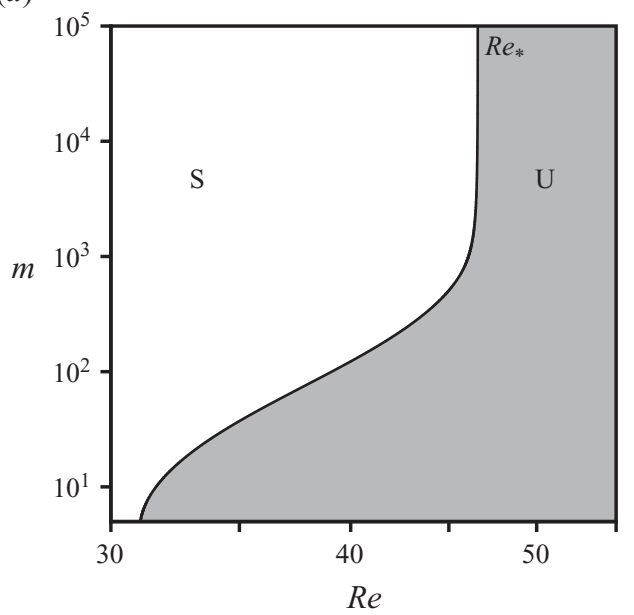

$1 / u_{r}$

(b)

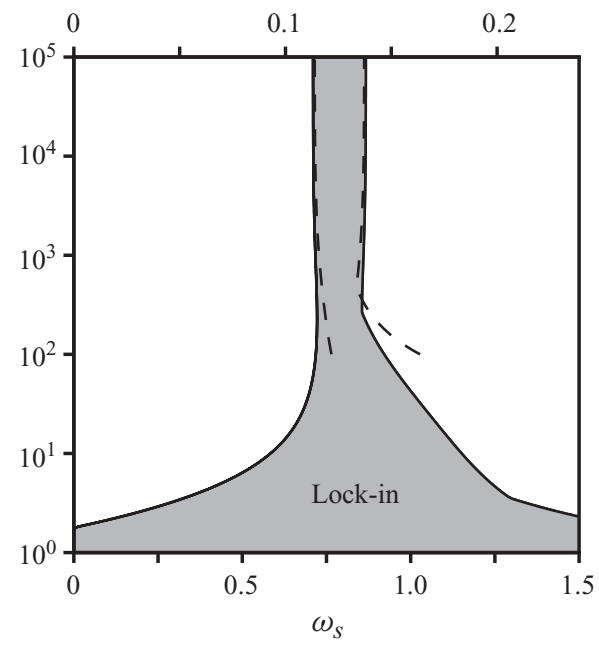

FIGURE 9. (a) Boundary separating the unstable domain from the stable domain in the plane $(R e, m)$ for $\gamma=0.01$ and $\omega_{s}=0.75$. (b) Boundary of the resonance width $\Delta \omega_{s}$ as a function of the mass ratio, for $\gamma=0.01$ and $R e=50$. The solid and dashed lines show the results obtained respectively using the extended model derived in $\S 6$ and the original model derived in $\S 3$.

by less than $5 \%$ for $m>150$. As discussed in $\S 5.2$, we find that the lock-in domain extends at low mass numbers, namely for $m \leqslant 300$ in the present case. If we further decrease the mass ratio, the lower bound of the lock-in domain reaches the zero value for $m=1.7$, although the magnitude of the cylinder displacement is now self-limited. When recast in terms of the reduced velocity $u_{r}$ defined by (3.11), this means that significant displacement amplitudes are expected even for arbitrarily large values of $u_{r}$. Such a result is thus reminiscent of that documented by Govardhan \& Williamson (2002), who reported the sudden onset of resonance over an infinitely large range of velocities below a so-called critical mass ratio $m_{c r i t}$. Although qualitative, the obtained value $m=1.7$ is in reasonable agreement with that reported by these authors, who predict a critical mass $m_{\text {crit }}=0.25$ for $R e=100$.

\section{Application to energy production through VIV}

The derivation of the extended model also allows to consider vortex-induced vibrations of a cylinder in water streams for renewable energy production, as discussed by Bernitsas et al. (2008). For instance, electrical energy will be produced if the oscillation of the cylinder displaces periodically a magnet within a coil. On the oscillator dynamics of the cylinder, the energy production device induces a structural damping term proportional to $\mathrm{d} y_{s} / \mathrm{d} t$, of the form used in (2.3). The linear oscillator equation governing the motion of the cylinder can be rewritten in the form of an energy equation as

$$
\frac{\mathrm{d} e}{\mathrm{~d} t}=-d(t)+p(t) .
$$

In (7.1), $e$ is the mechanical energy of the cylinder defined as the sum of its kinematic and potential energy:

$$
e=\frac{1}{2} \frac{m+c_{a}}{m}\left(\frac{\mathrm{d} y_{s}}{\mathrm{~d} t}\right)^{2}+\frac{1}{2} \omega_{s}^{2} y_{s}^{2}
$$


Similarly, $d$ is the energy dissipated by damping and $p$ is the energy extracted from the flow, respectively,

$$
d=2\left(\omega_{s} \gamma+\frac{2}{\pi m} \omega_{*} \zeta\right)\left(\frac{\mathrm{d} y_{s}}{\mathrm{~d} t}\right)^{2}, \quad p=\frac{2}{\pi m} c_{y} \frac{\mathrm{d} y_{s}}{\mathrm{~d} t} .
$$

Since the solution is periodic of period $T_{v}=2 \pi / \omega_{v}$ on the limit cycle, the mean mechanical energy averaged over a period is zero. In return, the mean energy lost by damping is equal to the mean energy extracted from the flow. From the perspective of energy production, one should note that the dissipated energy is determined by two contributions associated with structural and added damping, but that only the energy dissipated by structural damping is of practical interest. In contrast, the energy dissipated by the fluid cannot be recovered and is responsible for a loss of efficiency of the device. In practice, we obtain the leading-order energy dissipated by structural damping over a period by computing the time derivative of the cylinder position from system (3.25), along with the modified structural parameters (6.2), which yields

$$
\mathscr{D}=2 \omega_{s} \gamma \frac{1}{T_{v}} \int_{0}^{T_{v}}\left(\frac{\mathrm{d} y_{s}}{\mathrm{~d} t}\right)^{2} \mathrm{~d} t=4 \omega_{s} \omega_{*}^{2} \gamma|\mathscr{Y}|^{2} .
$$

In the following, the latter will also be referred to as the efficient mean energy that can be extracted from the flow.

We now set $m=10$, a mass ratio corresponding approximately to that of steel in water, and investigate the effect of structural damping on the magnitude of the mean energy $\mathscr{D}$. Results obtained using the extended model are presented in figure $10(a)$ for $\omega_{s}=0.8$ and $R e=50$. The energy tends to zero in the limit $\gamma \gg 1$, where the work received from the lift force is limited by the low amplitude of the cylinder displacement. It also tends to zero in the limit $\gamma \ll 1$, where the cylinder amplitude reaches an asymptotic limit, determined by the added damping force. Consequently, the energy reaches a maximum for a finite value of the structural damping $\gamma^{\max }$, defined by

$$
\mathscr{D}^{\max }\left(\omega_{s}, m, R e\right)=\max _{\gamma}\{\mathscr{D}\}=\mathscr{D}\left(\gamma^{\max }, \omega_{s}, m, R e\right),
$$

with $\gamma^{\max } \sim 0.13$ for the mass ratio and Reynolds numbers considered (vertical dotted line in figure $10 a$ ).

It is possible to determine similarly an optimal value of $\mathscr{D}^{\max }$ and $\gamma^{\max }$ for each natural frequency, keeping the mass ratio and the Reynolds number equal to $m=10, R e=50$. Results are displayed in figure $10(b)$ by the solid and dashed lines, respectively. The grey line corresponds to the value of the added damping term $2 \zeta / \pi m$, meaning that the damping force is dominated by the structural term in the resulting upper half-plane, and by the added damping term in the lower half-plane. The maximum efficient energy extracted from the flow increases with $\omega_{s}$ in the range $\omega_{s}<1$ : since the structural damping dominates, its magnitude can be simply increased by decreasing the value of $\gamma$. The structural coefficient drops to small values for $\omega_{s} \sim 1$, and the added damping term becomes dominant, which results in a saturation and a further decrease in the energy. Finally, the discontinuity at $\omega_{s} \sim 1.2$ corresponds to a transition from a three-real-roots to a one-real-root regime when solving for the phase shift equation. These results evidence the existence of a particular setting $\left(\gamma^{\text {opt }}, \omega_{s}^{\text {opt }}\right)$ for which the extracted energy reaches an optimum

$$
\mathscr{D}^{o p t}(m, R e)=\max _{\omega_{s}}\left\{\mathscr{D}^{m a x}\right\}=\mathscr{D}\left(\gamma^{o p t}, \omega_{s}^{o p t}, m, R e\right) .
$$



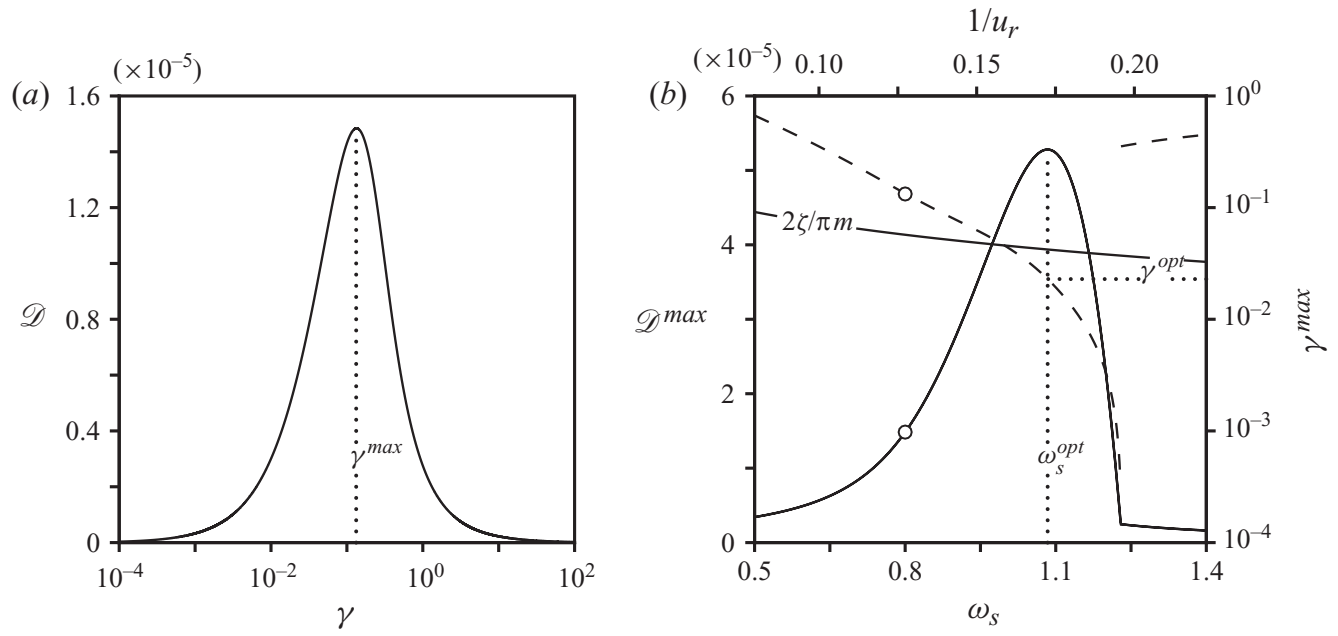

FIGURE 10. Effect of the structural parameters on the efficient mean energy $\mathscr{D}$ extracted from the flow for $m=10$ and $R e=50$. (a) Energy as a function of the structural damping for $\omega_{s}=0.8$. The vertical dotted line marks the value $\gamma^{\max }$ for which the maximum is reached. (b) Maximum energy $\mathscr{D}^{\max }=\mathscr{D}\left(\gamma^{\max }\right)$ as a function of the natural frequency $\omega_{s}$. The circles indicate the values extracted from $(a)$ for $\omega_{s}=0.8$. The horizontal solid line indicates the value of the added damping term $2 \zeta / \pi m$, whereas the vertical and lower horizontal dotted lines mark the optimal setting $\left(\omega_{s}^{o p t}, \gamma^{o p t}\right)$ for which the energy extracted from the flow is maximum.

For the mass ratio and Reynolds numbers considered, we obtain $\omega_{s}^{o p t}=1.08$ and $\gamma^{o p t}=0.023$, this setting defining precisely the 'tuning' at which the system should be operated to maximize the energy extracted from the free stream by an energy production device.

We have computed the vortex-shedding frequency $\omega_{v}\left(\gamma^{\max }\right)$ as a function of $\omega_{s}$, the results being shown in figure 11(a), along with the bisector of the plane $\left(\omega_{v}, \omega_{s}\right)$. Interestingly, we find that $\omega_{v}^{o p t}=\omega_{v}\left(\gamma^{\text {opt }}, \omega_{s}^{\text {opt }}\right)<\omega_{s}$, meaning that the cylinder oscillates at a frequency smaller than its natural frequency when the optimality condition is satisfied. Figure 11(b) finally presents the phase shift $\phi\left(\gamma^{\max }\right)$ similarly computed as a function of $\omega_{s}$. When the natural frequency is smaller than the nonlinear vortex-shedding frequency, the cylinder is in retarded half-quadrature with respect to the lift fluctuations, i.e. $\phi^{\text {opt }} \sim+\pi / 4$. When the natural frequency is larger than the vortex-shedding frequency, the cylinder is in advanced half-quadrature, i.e. $\phi_{\text {opt }} \sim-\pi / 4$. In contrast, the phase shift drops to small values when the natural frequency approaches that of vortex shedding, the cylinder and the lift fluctuations being nearly in phase at optimality.

\section{Discussion: connection with existing models}

Numerous models have been developed for the study of VIV at practically meaningful Reynolds numbers. From now on, we focus on the class of so-called wake oscillator models in which the wake dynamics is described by a single flow variable modelling the fluctuating nature of the vortex shedding (Birkoff \& Zarantanello 1957; Bishop \& Hassan 1964). This flow variable is assumed to satisfy a van der Pol or Rayleigh equation which models a self-sustained, stable and nearly harmonic oscillation of finite amplitude. In this section, we carry out the multiple time-scale analysis of the wake oscillator model, and compare the resulting set of equations to 

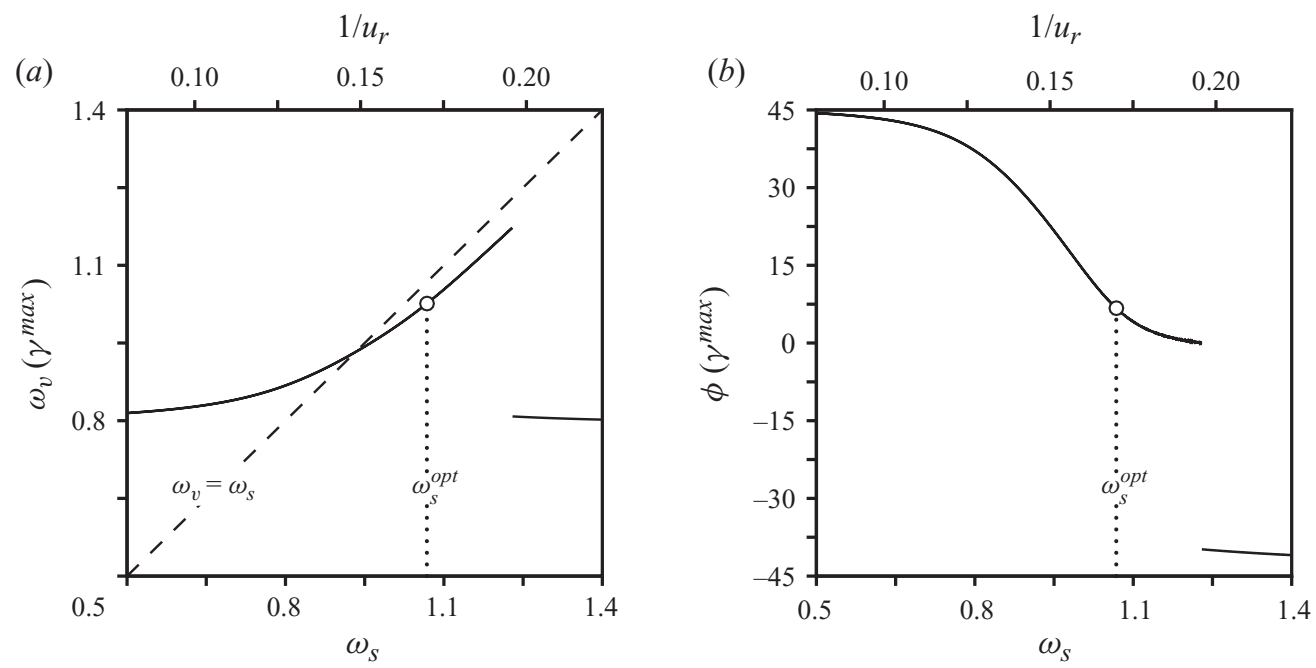

FIGURE 11. (a) Vortex-shedding frequency $\omega_{v}\left(\gamma^{\max }\right)$ and $(b)$ phase shift $\phi\left(\gamma^{\max }\right)$ as a function of $\omega_{s}$ for $m=10$ and $R e=50$. The vertical dotted line marks the optimal value $\omega_{s}^{o p t}$.

that issuing from the exact Navier-Stokes equations. It is worthwhile noting that such an expansion is exact close to threshold, and should be considered as only qualitative for higher Reynolds numbers.

\subsection{Flow model}

We consider an extension of the wake oscillator model based on a modified Duffingvan der Pol equation

$$
\frac{\mathrm{d}^{2} q}{\mathrm{~d} t^{2}}+2 \lambda_{r}\left(q^{2}-\epsilon^{2} \delta\right) \frac{\mathrm{d} q}{\mathrm{~d} t}+(\underbrace{2 \lambda_{i} \omega_{*} \epsilon^{2} \delta}_{\text {(i) }}-\underbrace{\frac{2}{3} \lambda_{r} \frac{\mu_{i}}{\mu_{r}} \omega_{*} q^{2}}_{\text {(ii) }}+\omega_{*}^{2}) q=f .
$$

This equation differs from the classical van der Pol equation by two terms noted (i) and (ii), accounting respectively for the linear and nonlinear frequency shifts of the saturated solution when the Reynolds number departs from threshold. As discussed by Facchinetti, de Langre \& Biolley (2004), the variable $q$ describing the wake oscillation should be defined as the lift magnification with respect to a fixed structure experiencing vortex shedding, i.e.

$$
q=2 \epsilon \sqrt{\delta} \frac{c_{y}}{\left|c_{y, f}\right|},
$$

where $\left|c_{y, f}\right|$ is the magnitude of the lift coefficient for a fixed cylinder. Note that the discrepancy with respect to the expression documented by Facchinetti et al. (2004) is due to a different normalization choice.

The right-hand side $f$ in (8.1) models the action of the cylinder motion on the wake oscillator, and various ad hoc forms have been postulated, including a displacement coupling (Krenk \& Nielsen 1999), a velocity coupling (Hartlen \& Currie 1970) and an acceleration coupling (Parkinson 1989) defined respectively as

$$
f=K y_{s} \text { (disp.), } f=K \frac{\mathrm{d} y_{s}}{\mathrm{~d} t} \text { (vel.), } f=K \frac{\mathrm{d}^{2} y_{s}}{\mathrm{~d} t^{2}} \text { (acc.), }
$$




$\begin{array}{cccc} & \text { Displacement } & \text { Velocity } & \text { Acceleration } \\ |\chi| & \frac{1}{2 \omega_{*}} K & \frac{1}{2} K & \frac{\omega_{*}}{2} K \\ \phi_{\chi} & \pi & -\frac{\pi}{2} & 0\end{array}$

TABLE 1. Modulus and phase of the $\chi$ coefficient for a displacement, a velocity and an acceleration coupling, as defined by (8.3).

$K \geqslant 0$ being a fixed parameter.

\subsection{Structure model}

For simplicity, we use here the rigorous asymptotic model derived in $\S 3$. Nevertheless, the approach can be generalized to account for the added forces, as has been done in $\S 6$. Assuming that the cylinder moves only in the transverse direction, the wake oscillator interacts with the linear oscillator equation

$$
\frac{\mathrm{d}^{2} y_{s}}{\mathrm{~d} t^{2}}+2 \omega_{s} \gamma \frac{\mathrm{d} y_{s}}{\mathrm{~d} t}+\omega_{s}^{2} y_{s}=s
$$

The right-hand-side term in (8.4) models the action of the fluid on the structure. For consistency with the Navier-Stokes equations, it must therefore satisfy

$$
s=\frac{2}{\pi m} c_{y}=\frac{2}{\pi m}\left|c_{y, f}\right| \frac{q}{2 \epsilon \sqrt{\delta}} .
$$

Since the lift force is proportional to the global mode of order $\epsilon$, we define here the leading-order lift coefficient $C_{y}$ such that $c_{y}=\epsilon C_{y}$ (respectively $c_{y, f}=\epsilon C_{y, f}$ ), and the coupling term finally reads as

$$
s=\frac{2}{\pi m}\left|C_{y, f}\right| \frac{q}{2 \sqrt{\delta}} .
$$

\subsection{Weakly nonlinear expansion}

The dimensionless wake variable $q$ can now be sought as

$$
q=\epsilon Q(T) \mathrm{e}^{\mathrm{i} \omega_{*} t}+\text { c.c. }
$$

the other scaling laws being identical to those defined in $\S 3$. The system of amplitude equations for $Q$ and $Y$ can be obtained by carrying out a very similar weakly nonlinear expansion. We obtain

$$
\begin{aligned}
& \frac{\mathrm{d} Q}{\mathrm{~d} T}=\lambda \delta Q-\lambda_{r}\left(1+\mathrm{i} \frac{\mu_{i}}{\mu_{r}}\right) Q|Q|^{2}+\mathrm{i} \chi Y, \\
& \frac{\mathrm{d} Y}{\mathrm{~d} T}=\omega_{*}(-\Gamma+\mathrm{i} \Omega) Y-\mathrm{i} \frac{\left|C_{y, f}\right|}{\pi \omega_{*} M} \frac{Q}{2 \sqrt{\delta}} .
\end{aligned}
$$

The retroaction of the structure onto the flow is modelled by the coefficient $\chi$ in $(8.8 a)$, whose modulus $|\chi|$ and phase $\phi_{\chi}$ depend on the chosen modelling, as summarized in table 1 . 


\subsection{Comparison and choice of the most appropriate model}

The lift coefficients of interest can be expressed simply in terms of the global mode amplitude as

$$
\begin{aligned}
c_{y}=\epsilon C_{y} & =-2 \epsilon \pi \beta|A| \sin \left(\omega_{*} t+\phi_{A}\right), \\
\left|c_{y, f}\right|=\epsilon\left|C_{y, f}\right| & =2 \epsilon \pi \beta\left|A_{f}\right|,
\end{aligned}
$$

where $\left|A_{f}\right|$ is the global mode amplitude for a fixed cylinder, i.e. $\left|A_{f}\right|=\sqrt{\lambda_{r} \delta / \mu_{r}}$. This allows to define simply the wake complex amplitude $Q$ as

$$
Q=\mathrm{i} \sqrt{\frac{\mu_{r}}{\lambda_{r}}} A .
$$

System of amplitude equations (8.8) can therefore be recast in terms of the amplitudes $A$ and $Y$. We now obtain

$$
\begin{aligned}
& \frac{\mathrm{d} A}{\mathrm{~d} T}=\lambda \delta A-\mu A|A|^{2}+\chi \sqrt{\frac{\lambda_{r}}{\mu_{r}}} Y, \\
& \frac{\mathrm{d} Y}{\mathrm{~d} T}=\omega_{*}(-\Gamma+\mathrm{i} \Omega) Y+\frac{\beta}{\omega_{*} M} A .
\end{aligned}
$$

The system (8.11) differs from (3.25) only by the coupling term in the fluid equation, so that the relevance of a displacement, velocity or acceleration coupling can be assessed by comparing the value of the exact phase $\phi_{\alpha}=\arg (\alpha)$ computed from the asymptotic analysis of the Navier-Stokes equations and that of the model phase $\phi_{\chi}=\arg (\chi)$. Since $\phi_{\alpha}=0.399$, this yields the following conclusions:

(a) Since $\cos \phi_{\alpha} \geqslant 0$, this value is consistent with the coupling coefficient predicted by a wake oscillator model, provided one uses either a pure acceleration coupling or an acceleration coupling combined with a displacement coupling of lower magnitude.

(b) Since $\sin \phi_{\alpha} \geqslant 0$, this value cannot be obtained by combining any of the couplings considered by Facchinetti et al. (2004). To reproduce the appropriate coefficient, one must use either an additional integral or a third-order derivative coupling, i.e.

$$
f=K \int y_{s} \mathrm{~d} t, \quad \text { or } \quad f=K \frac{\mathrm{d}^{3} y_{s}}{\mathrm{~d} t^{3}},
$$

or combine such a coupling with a velocity coupling of lower magnitude.

(c) Since $\cos \phi_{\alpha} \geqslant \sin \phi_{\alpha}$, the overall coupling is dominated by acceleration. Although the range of Reynolds number is not the same, these results are consistent with the conclusions of Facchinetti et al. (2004), who recommended using the acceleration model.

We now recall that the coupling coefficient $\alpha$ is determined by two distinct contributions associated with mass conservation and viscous effects. The latter term being weighted by the inverse of the Reynolds number, as seen from (3.20), it has been argued in previous studies that for sufficiently large Reynolds numbers, the viscous term should be neglected compared to the wall pressure $\hat{p}_{1 A}^{\dagger}$, implying that the coupling occurs only through the wall-normal component of the velocity (Marquet et al. 2009). This yields a new expression for the coupling coefficient, which is now 
defined by

$$
\begin{aligned}
\alpha \sim \mathscr{S}^{-1} \int_{\Upsilon_{w}} \mathrm{i} \omega_{*} \hat{p}_{1 A}^{\dagger} n_{y} \mathrm{~d} l & =0.00651+0.0528 \mathrm{i}, \\
\phi_{\alpha} & =1.448\left(82.9^{\circ}\right) .
\end{aligned}
$$

The results so obtained are qualitatively similar to those discussed from the lowReynolds-number case, as we obtain again $\cos \phi_{\alpha} \geqslant 0$ and $\sin \phi_{\alpha} \geqslant 0$. However, we now have $\cos \phi_{\alpha} \ll \sin \phi_{\alpha}$, meaning that the coupling is dominated by the integral or third-order term and no more by acceleration. Note that these conclusions are only qualitative, since the expansion procedure is meant for small departures from threshold. Moreover, when the flow becomes turbulent, the fluid viscosity should be replaced by an appropriate eddy viscosity, and the tangential velocity may play a significant role. Although this lies out of the scope of the present paper, the ability of such a model to reproduce the physics of VIV at large Reynolds numbers therefore deserves some further investigation.

\section{Conclusion}

In this study, we have considered the nonlinear dynamics of vortex-induced vibrations in the wake of a damped, spring-mounted, circular cylinder. A multiple time-scale expansion has been developed for Reynolds numbers close to the threshold of instability of the flow past a fixed cylinder, and the system of nonlinear equations governing the dynamics of the coupled flow-cylinder system has been derived. The key dominant balance considers a solid-to-fluid density ratio of order $\epsilon^{-4}$, the departure from criticality being classically assumed of order $\epsilon^{2}$. The cylinder displacement then occurs at order $\epsilon^{3}$ in the expansion and yields the coupling under the form of a thirdorder resonant boundary condition requiring an appropriate compatibility condition. All coefficients of the model have been analytically expressed as scalar products between a resonant forcing term arising at third order and the adjoint of the forced mode.

Analysing the linear part of the resulting dynamics has allowed to identify two modes of interest, namely a wake mode and a structure mode, the latter being responsible for the onset of subcritical vortex shedding at low solid-to-fluid density ratios. Analysing the nonlinear dynamics has demonstrated the ability of the present adjoint-based model to describe the main features of vortex-induced vibrations, in particular lock-in and hysteretic behaviours. We have shown that the cylinder motion is in phase with the lift fluctuations at lock-in, and either in advanced or retarded quadrature at lock-out. The exact model has been generalized to low mass ratios and mass-damping parameters, where the added-mass and added damping terms arising from the mass of displaced fluid must be taken into account. Using this extension, we have considered the impact of the damping parameter on the energy dissipated by the structure, which is of practical interest in the perspective of energy production, since it represents the efficient energy extracted from the flow by transfer to the cylinder motion. We have shown that there exists an optimal setting of the structural parameter for which the extracted energy is maximum, the cylinder motion being then in phase with the lift fluctuations.

Finally, we have discussed the connection between the present asymptotic, lowReynolds-number approach and the wake oscillator model used in the literature. We have shown that if the dynamics of the model is analysed close to threshold (which is by no way its domain of validity), it gives a system of amplitude equations 


$\begin{array}{cccccc} & n_{t} & \text { d.o.f. } & \lambda & \mu & \alpha \\ M_{1} & 117217 & 1053601 & 9.151+3.236 \mathrm{i} & 308.9-1025 \mathrm{i} & 0.03486+0.01477 \mathrm{i} \\ M_{2} & 180896 & 1626100 & 9.153+3.239 \mathrm{i} & 308.9-1025 \mathrm{i} & 0.03492+0.01472 \mathrm{i} \\ M_{3} & 293466 & 2638750 & 9.154+3.241 \mathrm{i} & 308.9-1025 \mathrm{i} & 0.03492+0.01472 \mathrm{i}\end{array}$

TABLE 2. Dependence of the model coefficients on different meshes differing by their spatial resolution. Here $n_{t}$ is the number of triangles and d.o.f. is the number of degrees of freedom for two-dimensional state vectors.

similar to the exact one presently computed. Comparing the exact and model coupling terms in the fluid equation shows that the effect of the structure on the fluid can be represented by a first coupling term proportional to the cylinder acceleration in the fluid equation, and by a second term that can originate either from an integral term or from a term proportional to the third derivative of the cylinder position. For low Reynolds numbers, this coupling is dominated by the contribution of acceleration, whereas the contribution of the integral or third-order term becomes dominant at high Reynolds numbers. Such results seem promising from the perspective of further investigating new high-Reynolds-number VIV models, for which no such coupling has been studied.

The authors are grateful to R. Violette, whose enthusiasm and fruitful comments have contributed to the present study.

\section{Appendix A. Sensitivity results to mesh spacing}

In order to assess convergence in the numerical results, the model coefficients have been computed for three meshes $M_{1}$ to $M_{3}$ differing by their spatial resolution, all results presented in the present study issuing from the intermediate mesh $M_{2}$. Results are detailed in table 2 for the values of $\lambda, \mu$ and $\alpha$ (the last coefficient $\beta$ being set to unity owing to the normalization of the global mode $\hat{\boldsymbol{q}}_{1 A}$ ), and show that nearly all constants are converged down to the third digit.

\section{Appendix B. Flow model and compatibility condition}

We detail here the derivation of the flow amplitude equation. As discussed in $\S 3.1$, we recall that the solution is identical to that computed from the fixed cylinder flow, up to order $\epsilon^{2}$. At order $\epsilon^{3}$, we find that the third-order solution $\boldsymbol{q}_{3}$ obeys the forced linear Navier-Stokes equations

$$
\boldsymbol{N} \partial_{t} \boldsymbol{q}_{3}+\boldsymbol{L}_{*} \boldsymbol{q}_{3}=\left(\hat{\boldsymbol{F}}_{3 r} \mathrm{e}^{\mathrm{i} \omega_{*} t}+\boldsymbol{F}_{3 n r}+\text { c.c., } 0\right)^{\mathrm{T}} .
$$

In (B 1), $\hat{\boldsymbol{F}}_{3 r}$ is a bulk resonant forcing term depending only from the lower-order solutions,

$$
\hat{\boldsymbol{F}}_{3 r}=-\frac{\mathrm{d} A}{\mathrm{~d} T} \hat{\boldsymbol{u}}_{1 A}+\delta A \hat{\boldsymbol{F}}_{3 A}+A|A|^{2} \hat{\boldsymbol{F}}_{3 A|A|^{2}},
$$

and $\boldsymbol{F}_{3 n r}$ is a non-resonant forcing term, not explicitly stated here for conciseness. The first resonant term of amplitude $\mathrm{d} A / \mathrm{d} T$ in (B 2) corresponds to the slow time 
evolution of the unknown amplitude $A$. The other resonant terms $\hat{\boldsymbol{F}}_{3 A}$ and $\hat{\boldsymbol{F}}_{3 A|A|^{2}}$ are

$$
\begin{aligned}
\hat{\boldsymbol{F}}_{3 A} & =-\nabla^{2} \hat{\boldsymbol{u}}_{1 A}-\nabla \hat{\boldsymbol{u}}_{1 A} \cdot \hat{\boldsymbol{u}}_{2 \delta}-\nabla \hat{\boldsymbol{u}}_{2 \delta} \cdot \hat{\boldsymbol{u}}_{1 A}, \\
\hat{\boldsymbol{F}}_{3 A|A|^{2}} & =-\nabla \hat{\boldsymbol{u}}_{1 A} \cdot \hat{\boldsymbol{u}}_{2|A|^{2}}-\nabla \hat{\boldsymbol{u}}_{2|A|^{2}} \cdot \hat{\boldsymbol{u}}_{1 A}-\overline{\nabla \hat{\boldsymbol{u}}_{1 A}} \cdot \hat{\boldsymbol{u}}_{2 A^{2}}-\nabla \hat{\boldsymbol{u}}_{2 A^{2}} \cdot \overline{\hat{\boldsymbol{u}}_{1 A}}
\end{aligned}
$$

The first term arises from the change in the diffusion when the Reynolds number varies, and the second term arises from the nonlinear interaction between the two contributions of the first-order solution together with the three contributions of the second-order solution. For further physical interpretations of these individual forcing terms, the reader is referred to Sipp \& Lebedev (2007).

The third-order solution $\boldsymbol{q}_{3}$ is sought as the superposition of a response to the non-resonant forcing term $\boldsymbol{F}_{3 n r}$ and a response to the resonant forcing term $\hat{\boldsymbol{F}}_{3 r}$, i.e.

$$
\boldsymbol{q}_{3}=\hat{\boldsymbol{q}}_{3 r} \mathrm{e}^{\mathrm{i} \omega_{*} t}+\boldsymbol{q}_{3 n r}+\text { c.c. }
$$

The response $\hat{\boldsymbol{q}}_{3 r}$ is thus the solution of the forced linear problem

$$
\left(\mathrm{i} \omega_{*} \boldsymbol{N}+\boldsymbol{L}_{*}\right) \hat{\boldsymbol{q}}_{3 r}=\left(\hat{\boldsymbol{F}}_{3 r}, 0\right)^{\mathrm{T}},
$$

along with the resonant condition $(3.8 b)$ at the cylinder wall, rewritten here as

$$
\hat{\boldsymbol{u}}_{3 r}=\boldsymbol{U}_{\boldsymbol{w}}+\boldsymbol{U}_{\boldsymbol{c}} \text {. }
$$

Since i $\omega_{*}$ is an eigenvalue for the problem (3.2), the operator i $\omega_{*} \boldsymbol{N}+\boldsymbol{L}_{*}$ cannot be inverted in (B 5). To avoid secular terms, or in other words, to be able to solve the expansion procedure at the third order, a compatibility condition has to be enforced.

We first consider the effect of bulk resonant terms only, as would be the case for a fixed cylinder. In this case, one uses a standard Fredholm alternative (Friedrichs 1973), specifying that the resonant forcing terms must be orthogonal to the kernel of the adjoint linearized Navier-Stokes operator, i.e.

$$
\int_{\Sigma} \hat{\boldsymbol{q}}_{1 A}^{\dagger} \cdot\left(\hat{\boldsymbol{F}}_{3 r}, 0\right)^{\mathrm{T}} \mathrm{d} x \mathrm{~d} y=0
$$

In (B 7), $\hat{\boldsymbol{q}}_{1 A}^{\dagger}$ is the adjoint global mode solution of the adjoint eigenvalue problem (3.16) at the critical Reynolds number $R e_{*}$. Owing to the form of the resonant forcing term (B 2), this yields the expression for the classical Stuart-Landau amplitude equation as

$$
\frac{\mathrm{d} A}{\mathrm{~d} T}=\lambda \delta A-\mu A|A|^{2}
$$

with $\lambda$ and $\mu$ being the complex coefficients defined from (3.18).

We now reintroduce the effect of the resonant boundary condition (B6). By construction of the adjoint operator $\boldsymbol{L}_{*}^{\dagger}$, we have, for any vector $\hat{\boldsymbol{q}}$ and $\hat{\boldsymbol{q}}^{\dagger}$ satisfying the direct and adjoint boundary conditions,

$$
\int_{\Sigma} \hat{\boldsymbol{q}}^{\dagger} \cdot\left(\boldsymbol{L}_{*} \hat{\boldsymbol{q}}\right) \mathrm{d} x \mathrm{~d} y=\int_{\Sigma}\left(\boldsymbol{L}_{*}^{\dagger} \hat{\boldsymbol{q}}^{\dagger}\right) \cdot \hat{\boldsymbol{q}} \mathrm{d} x \mathrm{~d} y .
$$

Owing to the resonant wall velocity (B 6), $\hat{\boldsymbol{q}}_{3 r}$ does not satisfy the direct boundary conditions, so the scalar product between the bulk resonant term and the adjoint 
global mode can be written as

$$
\begin{aligned}
\int_{\Sigma} \hat{\boldsymbol{q}}_{1 A}^{\dagger} \cdot\left(\hat{\boldsymbol{F}}_{3 r}, 0\right)^{\mathrm{T}} \mathrm{d} x \mathrm{~d} y & =\int_{\Sigma} \hat{\boldsymbol{q}}_{1 A}^{\dagger} \cdot\left(\mathrm{i} \omega_{*} \boldsymbol{N}+\boldsymbol{L}_{*}\right) \hat{\boldsymbol{q}}_{3 r} \mathrm{~d} x \mathrm{~d} y \\
& =\underbrace{\int_{\Sigma}\left(-\mathrm{i} \omega_{*} \boldsymbol{N}+\boldsymbol{L}_{*}^{\dagger}\right) \hat{\boldsymbol{q}}_{1 A}^{\dagger} \cdot \hat{\boldsymbol{q}}_{3 r} \mathrm{~d} x \mathrm{~d} y}_{=0}+\mathrm{BT},
\end{aligned}
$$

since $\boldsymbol{N}$ is diagonal, with BT being a non-zero boundary term arising during the integration by parts. As marked by the brace, the first term on the right-hand side of (B $10 b$ ) is zero, since $\hat{\boldsymbol{q}}_{1 A}^{\dagger}$ satisfies (3.16). A detailed calculation of the boundary term can be found in Giannetti \& Luchini (2007). We ultimately obtain

$$
\int_{\Sigma} \hat{\boldsymbol{q}}_{1 A}^{\dagger} \cdot\left(\hat{\boldsymbol{F}}_{3 r}, 0\right)^{\mathrm{T}} \mathrm{d} x \mathrm{~d} y=-\int_{\Upsilon_{w}}\left(\hat{p}_{1 A}^{\dagger} \boldsymbol{n}+\frac{1}{R \boldsymbol{e}_{*}} \nabla \hat{\boldsymbol{u}}_{1 A}^{\dagger} \cdot \boldsymbol{n}\right) \cdot\left(\boldsymbol{U}_{\boldsymbol{w}}+\boldsymbol{U}_{\boldsymbol{c}}\right) \mathrm{d} l,
$$

thus yielding the amplitude equation (3.17).

\section{REFERENCES}

BARKLEY, D. 2006 Linear analysis of the cylinder wake mean flow. Europhys. Lett. 75, 750-756.

Barkley, D., Gomes, M. G. M. \& Henderson, R. D. 2002 Three-dimensional instability in flow over a backward-facing step. J. Fluid Mech. 473, 167-190.

Bernitsas, M. M., Raghavan, K., Ben-Simon, Y. \& Garcia, E. M. H. 2008 Vivace (vortex induced vibration aquatic clean energy): a new concept in generation of clean and renewable energy from fluid flow. J. Offshore Mech. Arctic Engng 130, 041101(1-15).

Birkoff, G. \& Zarantanello, E. H. 1957 Jets, Wakes and Cavities. Academic Press.

Bishop, R. E. D. \& Hassan, A. Y. 1964 The lift and drag forces on a circular cylinder oscillating in a flowing fluid. Proc. R. Soc. Lond. A 277, 5175.

BLEvins, R. D. 1990 Flow-Induced Vibrations. Van Nostrand Reinhold.

Buffoni, E. 2003 Vortex shedding in subcritical conditions. Phys. Fluids 15, 814-816.

Cossu, C. \& Morino, L. 2000 On the instability of a spring-mounted circular cylinder in a viscous flow at low Reynolds numbers. J. Fluids Struct. 14, 183-196.

DAvis, T. A. 2004 A column pre-ordering strategy for the unsymmetric-pattern multifrontal method. ACM Trans. Math. Softw. 30 (2), 165-195.

Davis, T. A. \& Duff, I. S. 1997 An unsymmetric-pattern multifrontal method for sparse LU factorization. SIAM J. Matrix Anal. Appl. 18 (1), 140-158.

Ehrenstein, U. \& Gallaire, F. 2005 On two-dimensional temporal modes in spatially evolving open flows: the flat-plate boundary layer. J. Fluid Mech. 536, 209-218.

FACChinetTi, M. L., DE LANGRE, E. \& Biolley, F. 2004 Coupling of structure and wake oscillators in vortex-induced vibrations. J. Fluids Struct. 19, 123-140.

FRIEDRICHS, K. O. 1973 Spectral Theory of Operators in Hilbert Space. Springer.

GabBaI, R. D. \& Benaroya, H. 2005 An overview of modeling and experiments of vortex-induced vibration of circular cylinders. J. Sound Vib. 282, 575-616.

Giannetti, F. \& Luchini, P. 2007 Structural sensitivity of the first instability of the cylinder wake. J. Fluid Mech. 581, 167-197.

Govardhan, R. \& Williamson, C. H. K. 2000 Modes of vortex formation and frequency response of a freely vibrating cylinder. J. Fluid Mech. 420, 85-130.

Govardhan, R. \& Williamson, C. H. K. 2002 Resonance forever: existence of a critical mass and an infinite regime of resonance in vortex-induced vibration. J. Fluid Mech. 473, 147-166.

Hartlen, R. T. \& Currie, I. G. 1970 Lift-oscillator model of vortex-induced vibration. J. Engng Mech. Div. 96, 577-591.

JACKSON, C. P. 1987 A finite-element study of the onset of vortex shedding in flow past variously shaped bodies. J. Fluid Mech. 182, 23-45.

Krenk, S. \& Nielsen, S. R. K. 1999 Energy balanced double oscillator model for vortex-induced vibrations. J. Engng Mech. 125, 263-271. 
LANDAU, L. D. \& Lifshitz, E. M. 1976 Mechanics, vol. 1, 3rd ed. Butterworth-Heinenann.

DE LANGRE, E. 2006 Frequency lock-in is caused by coupled-mode flutter. J. Fluids Struct. 22, 783-791.

Marquet, O., Lombardi, M., ChomaZ, J.-M., SipP, D. \& JacQuin, L. 2009 Direct and adjoint global modes of a recirculation bubble: lift-up and convective non-normalities. J. Fluid Mech. 622, $1-21$.

Mathis, C., Provansal, M. \& Boyer, L. 1984 Bénard von-Kármán instability: an experimental study near the threshold. J. Phys. Lett. Paris 45, 483-491.

MitTAL, S. \& Singh, S. 2005 Vortex-induced vibrations at subcritical Re. J. Fluid Mech. 534, 185-194.

Parkinson, G. 1989 Phenomena and modeling of flow-induced vibrations of bluff bodies. Prog. Aerosp. Sci. 26, 169-224.

Provansal, M., Mathis, C. \& Boyer, L. 1987 Bénard von-Kármán instability: transient and forced regimes. J. Fluid Mech. 182, 1-22.

RamberG, S. E. \& Griffin, O. M. 1981 Hydrodynamics in ocean engineering. In Hydroelastic Response of Marine Cables and Risers, pp. 1223-1245. Norwegian Institute of Technology, Trondheim, Norway.

SarpKaYA, T. 1978 Fluid forces on oscillating cylinders. ASCE J. Waterway Port Coastal Ocean Div. 104, 275-290.

SARPKaYA, T. 2004 A critical review of the intrinsic nature of vortex-induced vibrations. J. Fluids Struct. 19, 389-447.

Schmid, P. J. \& Henningson, D. S. 2001 Stability and Transition in Shear Flows. Springer.

SipP, D. \& LebedeV, A. 2007 Global stability of base and mean flows: a general approach and its applications to cylinder and open cavity flows. J. Fluid Mech. 593, 333-358.

Skop, R. A. \& Balasubramanian, S. 1997 A new twist on an old model for vortex-induced vibrations. J. Fluids Struct. 11, 395-412.

Tezduyar, T. E., Behr, M. \& Liou, J. 1992a A new strategy for finite element computations involving moving boundaries and interfaces the deforming-spatial-domain/space-time procedure. I. The concept and the preliminary tests. Comput. Meth. Appl. Mech. Engng 94, 339-351.

Tezduyar, T. E., Behr, M., Mittal, S. \& Liou, J. $1992 b$ A new strategy for finite element computations involving moving boundaries and interfaces the deforming-spatialdomain/space-time procedure. II. Computation of free-surface flows, two-liquid flows, and flows with drifting cylinders. Comput. Meth. Appl. Mech. Engng 94, 353-371.

Williamson, C. H. K. \& Govardhan, R. 2004 Vortex-induced vibrations. Annu. Rev. Fluid Mech. 36, 413-455. 